\title{
Cross-sectional imaging findings of splenic infections: is differential diagnosis possible?
}

\author{
Ali Devrim Karaosmanoglu ${ }^{1}$ - Aycan Uysal ${ }^{2} \cdot$ Omer Onder $^{1} \cdot$ Peter F. Hahn ${ }^{3}$. Deniz Akata ${ }^{1} \cdot$ Mustafa Nasuh Ozmen ${ }^{1}$. \\ Musturay Karcaaltıncaba ${ }^{1}$
}

Received: 11 April 2021 / Revised: 12 May 2021 / Accepted: 19 May 2021 / Published online: 28 May 2021

○ The Author(s), under exclusive licence to Springer Science+Business Media, LLC, part of Springer Nature 2021

\begin{abstract}
The spleen plays an important role in the immunological homeostasis of the body. Several neoplastic and non-neoplastic diseases may affect this organ, and imaging is of fundamental importance for diagnosis. Infectious diseases of the spleen can be encountered in daily radiology practice, and differential diagnosis may sometimes be challenging. Infectious involvement of the spleen can be primary or secondary to a different source outside the spleen. Despite the fact that different infectious diseases may cause similar imaging findings, we believe that differential diagnosis between different causes may also be possible in certain patients with imaging. Early diagnosis may potentially enhance patients' treatment and outcome. In this review, we aimed to increase imaging specialists' awareness of splenic infections by describing the multimodality imaging features of common and atypical infections of the spleen with their differential diagnoses.
\end{abstract}

Keywords Spleen $\cdot$ İnfection $\cdot$ İmaging $\cdot$ Differential diagnosis $\cdot$ Cross-sectional

$\begin{array}{ll}\text { Abbreviations } & \\ \text { US } & \text { Ultrasound } \\ \text { CT } & \text { Computed tomography } \\ \text { MRI } & \text { Magnetic resonance imaging } \\ \text { GRE } & \text { Gradient-echo } \\ \text { T1W, T2W, T2*W } & \text { T1-weighted, T2-weighted, } \\ & \text { T2*-weighted } \\ \text { DWI } & \text { Diffusion-weighted imaging } \\ \text { IPT } & \text { Inflammatory pseudotumor } \\ \text { TB } & \text { Tuberculosis } \\ \text { CSD } & \text { Cat scratch disease } \\ \text { MAC } & \text { Mycobacterium avium-intracellulare } \\ & \text { complex } \\ \text { EMH } & \text { Extramedullary hematopoiesis } \\ \text { SANT } & \text { Sclerosing angiomatoid nodular } \\ & \text { transformation }\end{array}$

Ali Devrim Karaosmanoglu

alidevrim76@yahoo.com

1 Department of Radiology, Hacettepe University School of Medicine, Ankara 06100, Turkey

2 Department of Radiology, Gulhane Training and Research Hospital, Ankara 06010, Turkey

3 Department of Radiology, Massachusetts General Hospital, Harvard Medical School, Boston, MA 02114, USA
MIS-C

SLE

Multisystem inflammatory syndrome in children

Systemic lupus erythematosus

\section{Introduction}

Imaging plays an essential role in the diagnosis of splenic diseases. The spleen is a well-known infection site, and diagnosis may be challenging due to overlapping imaging features of different diseases. Imaging features of splenic infections are mostly non-specific, and there are imaging similarities between other neoplastic or non-infectious causes. Therefore, obtaining adequate clinical history is important for correct diagnosis. Serologic tests may also be helpful in certain diseases. In certain patients, image-guided (US or rarely CT) percutaneous biopsy might be necessary for a definitive diagnosis [1,2].

In this article, we will begin with the main imaging modalities used to evaluate the spleen and then move on to the radiological and clinical features of different infectious diseases affecting the spleen. Finally, we will review splenic involvement patterns in infectious diseases and their differential diagnoses. 


\section{Imaging of the spleen}

The spleen is frequently involved in a variety of diseases, including but not limited to congenital anomalies, vascular diseases, infectious/inflammatory diseases, benign and malignant neoplastic disorders, and metabolic diseases. Despite its common involvement in different diseases, radiologic referrals for splenic abnormalities are less common in daily practice compared to other abdominal organs [3].

Ultrasound (US), computed tomography (CT), and magnetic resonance imaging (MRI) may all be effectively used to assess the spleen. Complementary use of different imaging modalities in the same patient may help overcome the shortcomings of individual imaging modalities.
Although evaluation of the spleen tends to be institutional-dependent, US is generally the first-line imaging modality [3]. Even though operator dependence and patients' body habitus are the main limitations of US, color Doppler mode may help evaluate certain patients. As it can provide real-time images, US may also be effectively used for percutaneous biopsy of splenic lesions [4]. Additionally, in some cases, US may better demonstrate splenic lesions than the other modalities (Figs. 1 and 2). Despite all its advantages, US is limited in lesion characterization. Splenic lesions typically have a non-specific appearance of hypoechoic nodules in most examinations.

CT, along with US, is an important imaging modality with widespread use for imaging of the spleen. Contrastenhanced CT images in the portal venous phase are preferred for lesion characterization. CT may contribute to the
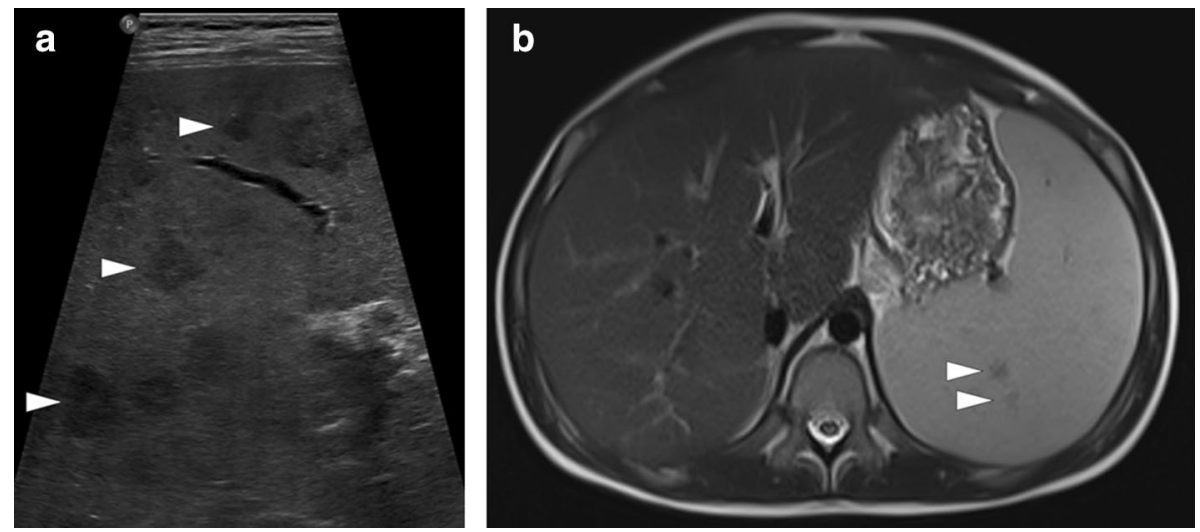

Fig. 1 Hemophagocytic lymphohistiocytosis: 12-year-old male patient presented with high fever, diffuse rash, and jaundice. Physical examination revealed abdominal tenderness and hepatosplenomegaly. The patient was diagnosed with hemophagocytic lymphohistiocytosis after an extensive clinical and laboratory workup. a Gray-scale US image demonstrated multiple hypoechoic nodular lesions of varying sizes in the spleen (arrowheads). b Axial plane T2W and postcontrast T1W (not shown) abdominal MR images obtained the next day after the initial US examination showed only a few of the sonographically detected lesions (arrowheads)

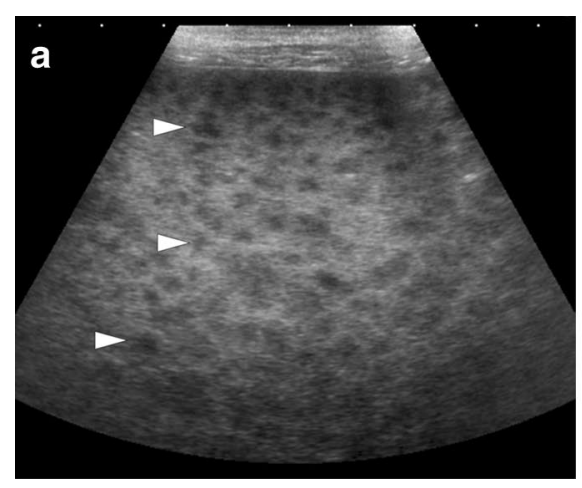

Fig. 2 Diffuse large B-cell lymphoma: 54-year-old female presented with unintentional weight loss and night sweats. Physical examination revealed massive splenomegaly and enlarged axillary lymph nodes. a Gray-scale US image demonstrated multiple subcentimeter hypoechoic nodules scattered throughout the splenic parenchyma (arrow-

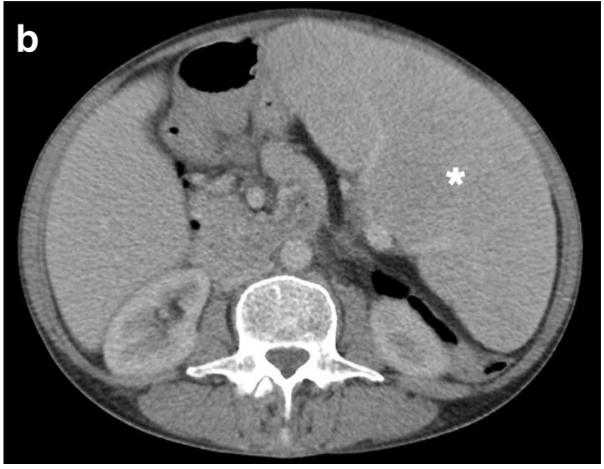

heads). b Axial plane postcontrast abdominal CT performed the next day after the initial US examination showed only massive splenomegaly (asterisk) with no discernible parenchymal nodules. US-guided percutaneous biopsy confirmed the diagnosis of lymphoma 
evaluation of the margins, density, and presence of solid component of the splenic lesions [5].

While certain features of splenic lesions may be better characterized with CT (e.g., calcifications), MRI can be more helpful for lesion characterization with its high softtissue resolution and its unique ability for potential tissue characterization with various imaging sequences [6]. It can be used as a problem solver in equivocal cases that cannot be characterized with US and CT and in cases where the malignancy cannot be excluded.

MRI pulse sequences used for spleen evaluation do not differ significantly from sequences used for standard abdominal MRI. Basic sequences include axial and coronal plane T2-weighted (T2W) turbo/fast spin-echo imaging, axial plane gradient-echo (GRE) breath-hold T1-weighted (T1W) in-phase and out-of-phase chemical shift imaging, axial plane three-dimensional GRE breath-hold pre-contrast and dynamic contrast-enhanced imaging [3, 7].

Although axial GRE T2*-weighted $(\mathrm{T} 2 * \mathrm{~W})$ imaging sequence is helpful in detecting the presence of hemosiderin, its use in spleen evaluation is optional [3]. In and out of phase $\mathrm{T} 1 \mathrm{~W}$ images may also provide information about iron accumulation in certain diseases which may manifest with focal siderotic lesions. This type of lesion is expected to have low signal in both $\mathrm{T} 1$ and $\mathrm{T} 2 \mathrm{~W}$ images $[7,8]$.

Since the spleen is an organ that physiologically restricts diffusion, the use of diffusion-weighted imaging (DWI) is limited. However, it can be beneficial in some clinical situations, such as investigating the accessory spleen [9]. Although there are publications in the literature that DWI can contribute to conventional sequences in the differentiation of malignant and benign splenic lesions, there is no consensus yet. It has been stated that in addition to malignant lesions, restricted diffusion can be observed in abscesses. On the other hand, DWI may not be reliable in hemorrhagic lesions due to the magnetic susceptibility effect secondary to the blood products [10].

On post-contrast imaging with CT and MR, different imaging pitfalls may be observed on dynamic imaging due to the unique blood flow pattern of the spleen. Therefore, it is of critical importance to know these pitfalls and avoid them to prevent false diagnoses. The most well-known of these pitfalls is the so-called "zebra pattern", which is characteristically observed in the early phases. In the early arterial phase, pseudonodular or pseudoinfiltrative lesion appearances may also be seen. All these pseudolesion appearances tend to disappear in the late venous phase with complete homogenization of the splenic parenchyma (Fig. 3) [3].

\section{Splenic infectious diseases}

Splenic infections are uncommon clinical entities, and therefore, neither imagers nor clinicians may be well-experienced to diagnose these diseases. Adding more complexity to this situation, clinical features, general laboratory tests, and imaging findings are mostly non-specific.

According to the causative organisms, splenic infectious diseases can be classified as viral, bacterial, parasitic, and opportunistic infections. A comprehensive summary of clinical and radiological findings of splenic infections can be found in Table 1.
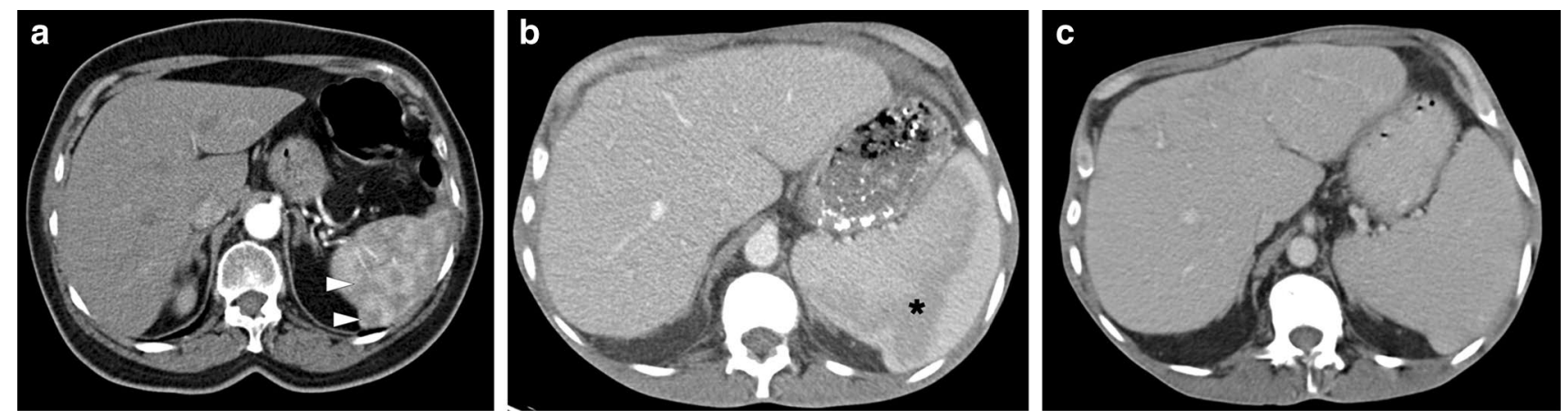

Fig. 3 Importance of accurate imaging phase in two different patients: a 64-year-old female patient with fever of unknown origin and weight loss. Axial plane postcontrast abdominal CT image showed multiple hypodense nodules in the spleen (arrowheads) due to the inappropriate imaging phase. This pseudonodular appearance completely disappeared on the repeat CT scan performed in the portal venous phase (not shown). b 44-year-old male patient with chronic kidney disease presented with abdominal pain. Axial plane postcontrast abdominal CT showed hypodense infiltrative appearance in the spleen (asterisk), thought to be suspicious for lymphoproliferative disease involvement. c Abdominal CT of the same patient in (b) performed 18 hours after the first CT scan. Control abdominal CT image in a later phase demonstrated homogenization of the spleen parenchyma with no apparent lesion 


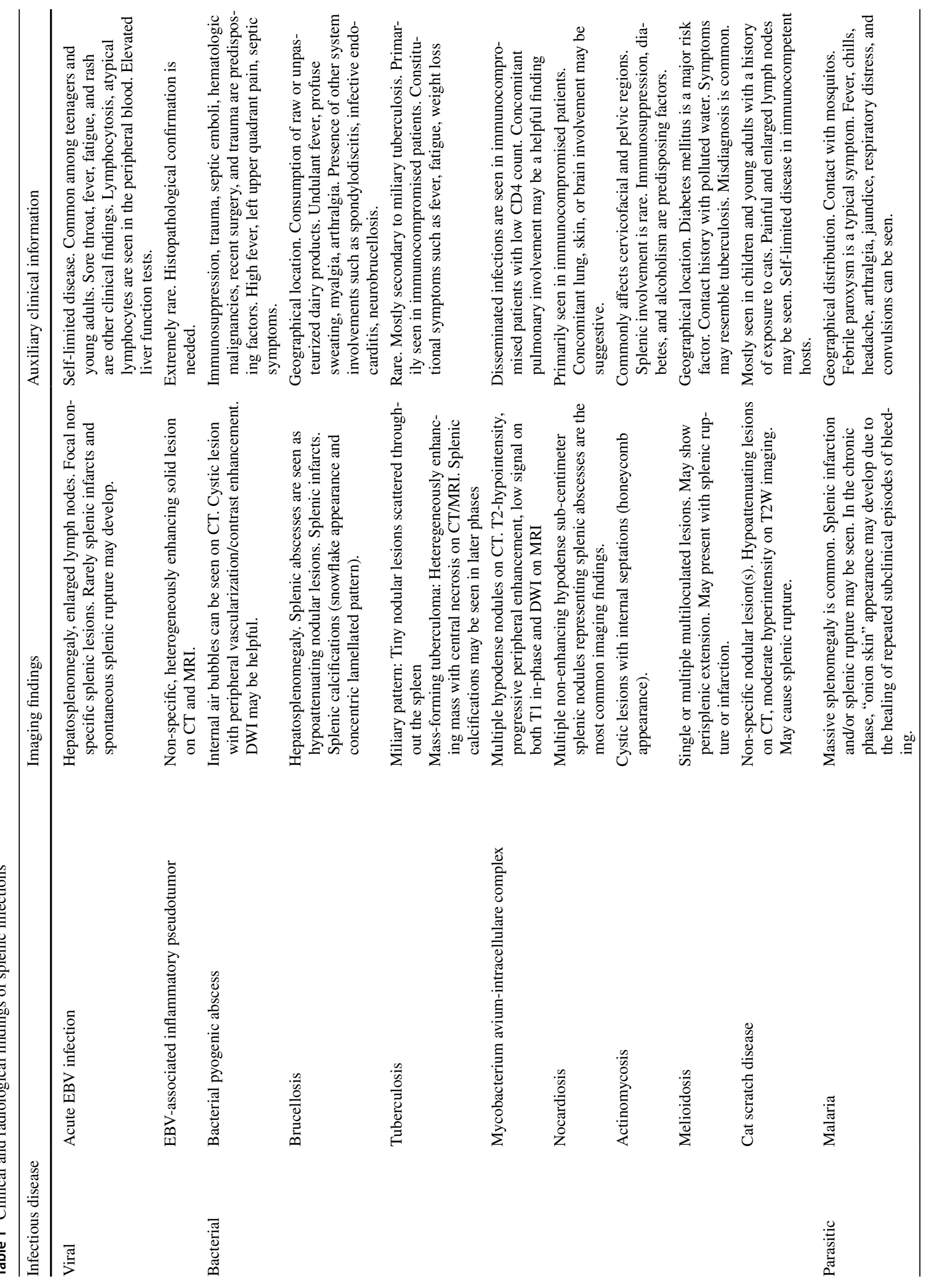




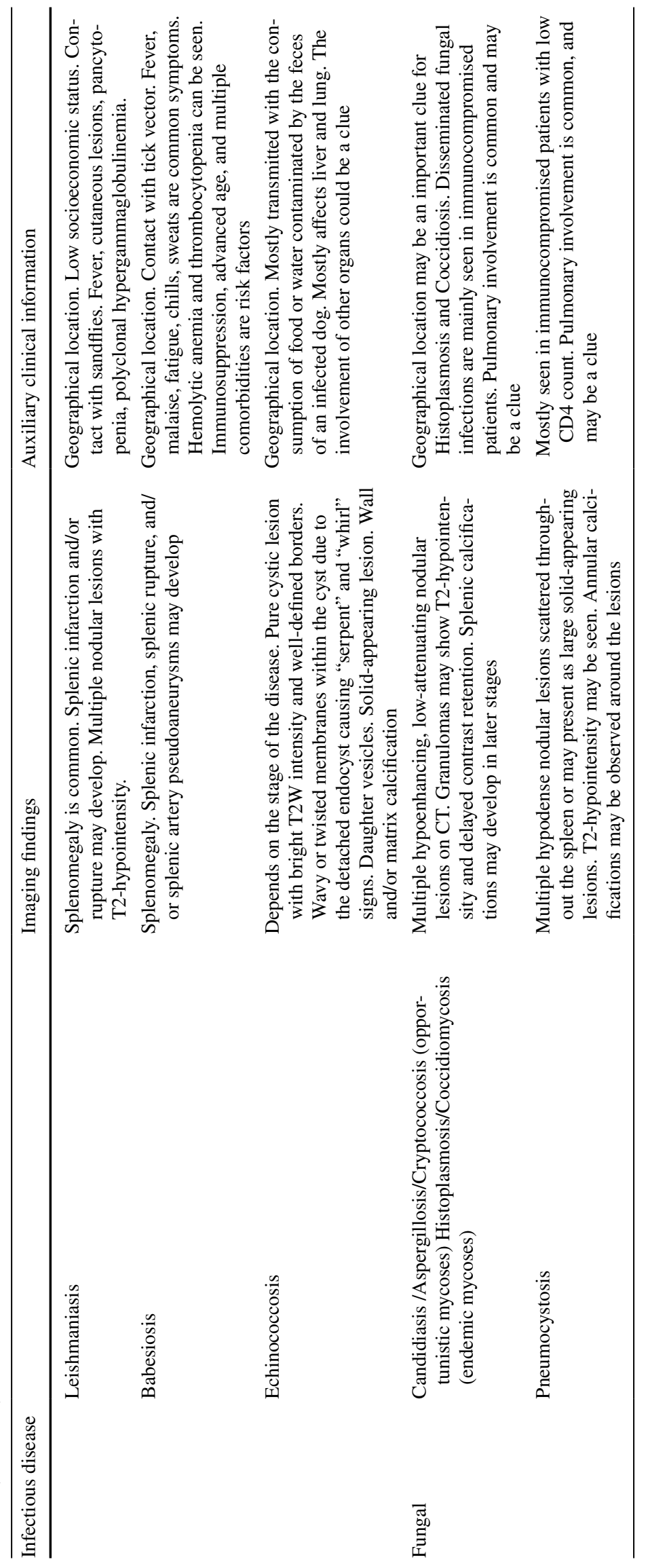




\section{Viral infections}

\section{Acute EBV infection and EBV-associated inflammatory}

Pseudotumor of the spleen Infectious mononucleosis is a common viral infection caused by EBV. Hepatosplenomegaly, rash, enlarged lymph nodes, sore throat, fever, and fatigue are among the common symptoms. Splenic infarct and spontaneous rupture are rare events that may happen during the course of the disease. On CT and MRI, splenic infarctions, regardless of the etiology, typically appear as wedge-shaped non-enhancing areas within the spleen, typically in subcapsular location [11]. The size of the infarcts can be variable, but diagnosis is typically straightforward in the clinical context. Splenic rupture has been reported in less than $0.5 \%$ of the cases [12]. The mechanism of splenic rupture is not well understood. On imaging, splenomegaly with loss of capsular integrity and associated perisplenic fluid are the most common imaging findings of acute rupture.

There are also publications reporting focal splenic lesions in the acute phase of the disease. Lymphoid hyperplasia and associated splenic infarcts may present as focal nodular lesions in these patients (Fig. 4) [13, 14].

EBV-associated inflammatory pseudotumors (IPTs) are extremely rare, with only sporadic cases reported. Histopathologically, splenic IPTs are typically characterized by spindle cell proliferation detected by specific immunohistochemical markers [15]. EBV is one of the etiologic factors for splenic IPTs in addition to autoimmune, reactive, and neoplastic causes. The most commonly encountered group of splenic IPT related to EBV infection is IPT-like follicular dendritic cell tumor [16]. Meanwhile, it should be borne in mind that Hodgkin lymphoma may also be associated with EBV infection. EBV-related malignant lymphoproliferative disease should also be considered in differential diagnosis, especially in immunocompromised patients (Fig. 5) [17].

In cases of splenic IPTs, most patients present with B symptoms, and clinically lymphoma may be considered as the primary diagnosis. On CT, the imaging findings are non-specific, and lymphoma is usually the leading diagnosis. These lesions appear as solid lesions of variable contrast enhancement on CT and MR studies (Fig. 6). This enhancement pattern is typically heterogeneous, and primary splenic malignant tumors should also be considered in the differential diagnosis [14]. Histopathological confirmation is almost always needed for confirmation. Clinically recurrence is rare, and the prognosis is generally benign [16].

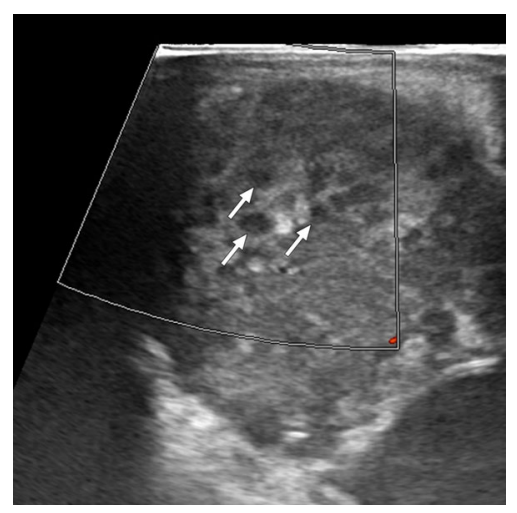

Fig. 5 EBV-associated lymphoproliferative disease: 2-year-old boy presented with fever, rash, and intermittent diarrhea. Physical examination revealed severe hepatosplenomegaly. Abdominal US was subsequently performed. Gray-scale US image demonstrated multiple subcentimeter hypoechoic nodules scattered throughout the hepatic (not shown) and splenic parenchymas with no prominent vascularity on color flow Doppler US (arrows). Histopathologic examination of the percutaneously biopsied liver lesions confirmed EBV-associated high-grade B-cell lymphoma
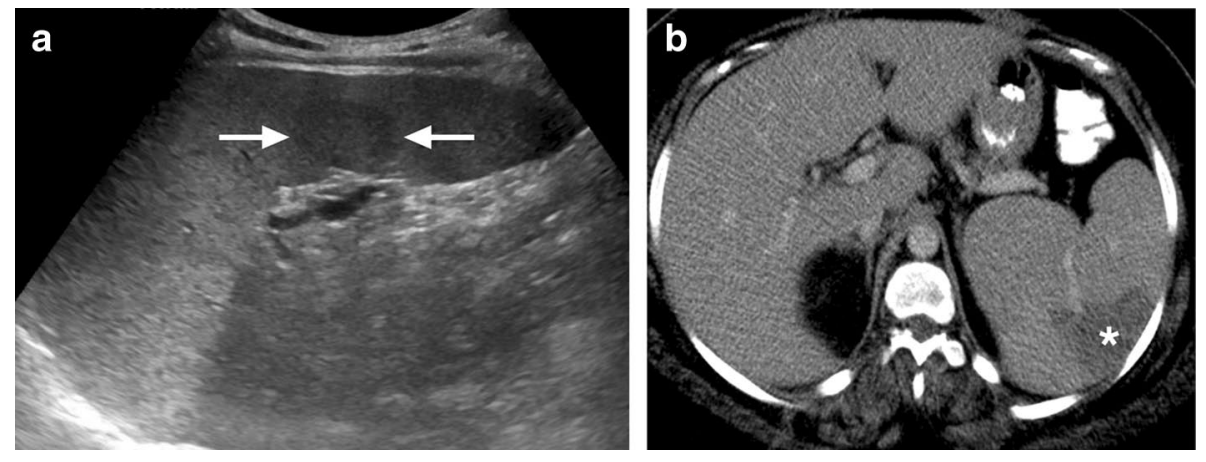

Fig. 4 Acute Epstein-Barr virus (EBV) infection in two different patients: a 24-year-old male patient with serologically and clinically proven EBV infection. Gray-scale US image showed a hypoechoic solid mass with well-defined borders in the upper pole of the spleen (arrows). Post-treatment follow-up imaging two weeks after the initial study showed the complete disappearance of this lesion (not shown). b 29-year-old female with no past medical history presented with left upper quadrant pain and fever. The serologic evaluation confirmed acute EBV infection. Axial plane postcontrast abdominal CT image demonstrated splenomegaly and peripherally located wedge-shaped hypodense area consistent with splenic infarct (asterisk) 

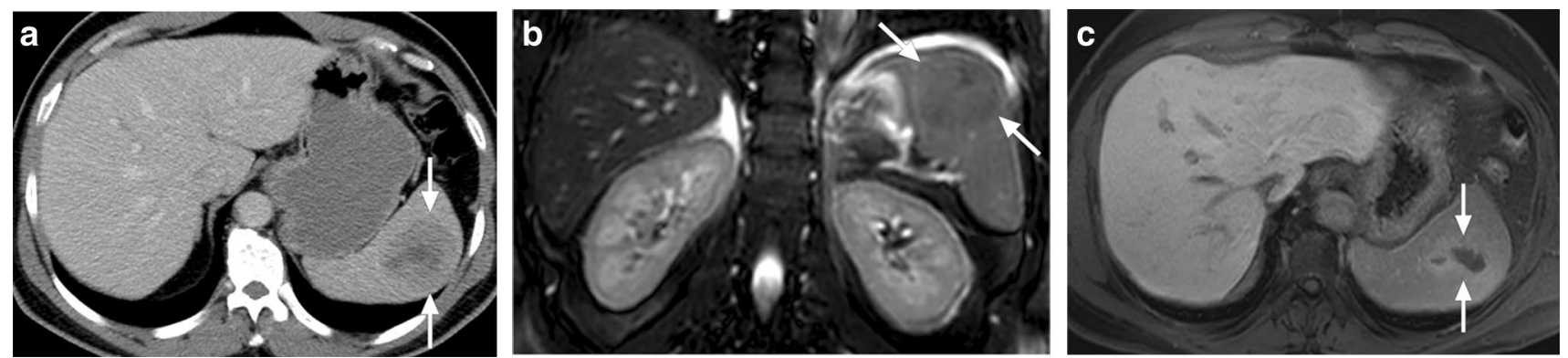

Fig. 6 EBV-associated inflammatory pseudotumor: 45-year-old male with no significant past medical history presented with mild left upper quadrant pain. US study showed a splenic mass (not shown). a Axial postcontrast abdominal CT image showed a heterogeneously-enhancing solid mass with central cystic/necrotic component (arrows). b Coronal plane T2W image showed a hypo-isointense solid

\section{Bacterial infections}

\section{Splenic pyogenic abscess}

Splenic microbial abscesses are uncommon clinical conditions with incidence reported to be between 0.2 and $0.7 \%$ in autopsy series [18]. Immunosuppression, trauma, septic emboli, hematologic malignancies, recent surgery, and trauma are common predisposing conditions. Bacteria are the most common etiologic agents, but rarely fungi and protozoa may also cause splenic abscesses. In the majority of abscesses, streptococci or staphylococci are present, and around 50\% of the patients' blood cultures were found to be positive [19]. These abscesses typically appear as hypodense lesions on CT with central fluid attenuation and peripheral contrast enhancement (Fig. 7). On MRI, they are mostly seen as T2-hyperintense lesions with peripheral contrast enhancement. However, their T1W and T2W signal intensities can vary depending on the content. Although its use in the spleen is limited, DWI may also be helpful [20].

Antibiotic therapy and splenectomy were conventional treatment approaches, but percutaneous drainage under imaging guidance has been applied in the last two decades, with successful results. Unilocular or paucilocular collections are more responsive to percutaneous drainage, whereas multiloculated collections are less conducive to successful percutaneous drainage [21]. In the presence of a pyogenic splenic abscess without obvious etiology, it may be helpful to think about and investigate bacterial endocarditis as a possible source of septic emboli.

\section{Brucellosis}

Brucellosis is a zoonosis that mostly has an insidious clinical course with multisystemic involvement. It is more common in the Mediterranean and Middle Eastern countries. mass in the splenic parenchyma (arrows). c Axial plane T1W postcontrast image showed heterogeneous contrast enhancement (arrows). As the imaging findings were not found to be conclusive for a benign process, the patient underwent splenectomy. Final histopathological examination confirmed EBV-related inflammatory pseudotumor

Spondylodiscitis, osteomyelitis, infective endocarditis, pneumonia, epididymoorchitis, and neurobrucellosis may all be seen in the course of the disease [22]. Hepatosplenomegaly, hepatitis, and splenic brucellosis may all be observed in abdominal involvement.

In acute hepatosplenic brucellosis, hepatosplenomegaly and elevated liver function tests are common. In patients with chronic splenic infection, splenic abscesses and calcifications may be observed. Splenic abscesses are generally seen in infections with B. melitensis and B. suis species. On $\mathrm{CT}$, they were reported to appear as hypoattenuating nodular lesions (Fig. 8). Rare cases of splenic infarcts in the course of splenic brucellosis have also been reported [23, 24].

Peripheral or central gross calcifications with a snowflake appearance and less commonly concentric lamellated pattern of splenic calcifications have been described in chronic splenic brucellomas [24]. Serological tests and culture are needed for confirmation, even if clinical history and radiological findings suggest brucellosis.

\section{Tuberculosis}

Tuberculosis (TB) is still a major health problem, despite all the developments in diagnosis and treatment, in certain parts of the world. Lungs are the most commonly involved organs, but extrapulmonary involvement is seen in around 15\%-20\% of all cases [25]. Splenic involvement is an uncommon presentation. The symptoms are mostly non-specific, with fever being the most common (in $82 \%$ ), followed by fatigue, weight loss, and splenomegaly [26]. HIV infection and other causes for immunosuppression may facilitate splenic TB, and it is extremely rare in immunocompetent patients [27]. Splenic involvement may appear as a solitary mass, multiple nodular granulomas, or miliary pattern (Fig. 9).

In patients with mass-forming tuberculomas, the differential diagnosis from neoplastic causes may be extremely 
b
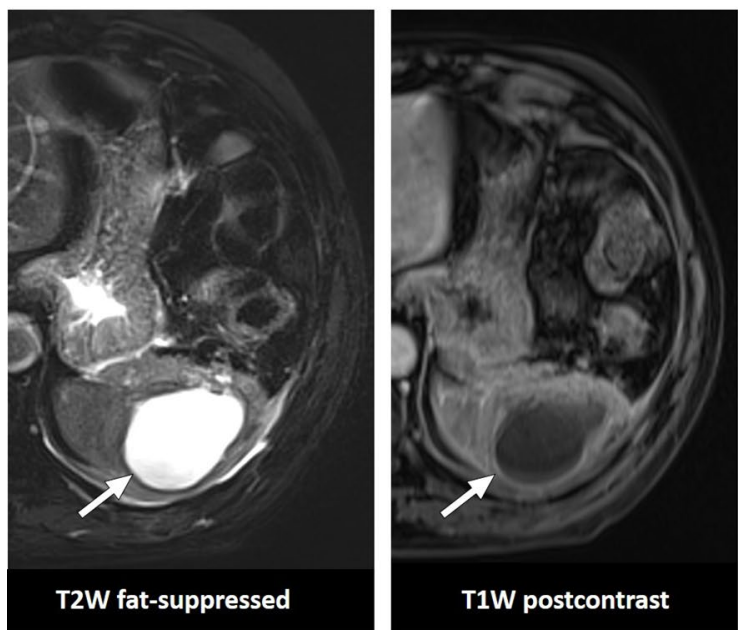

T1W postcontrast
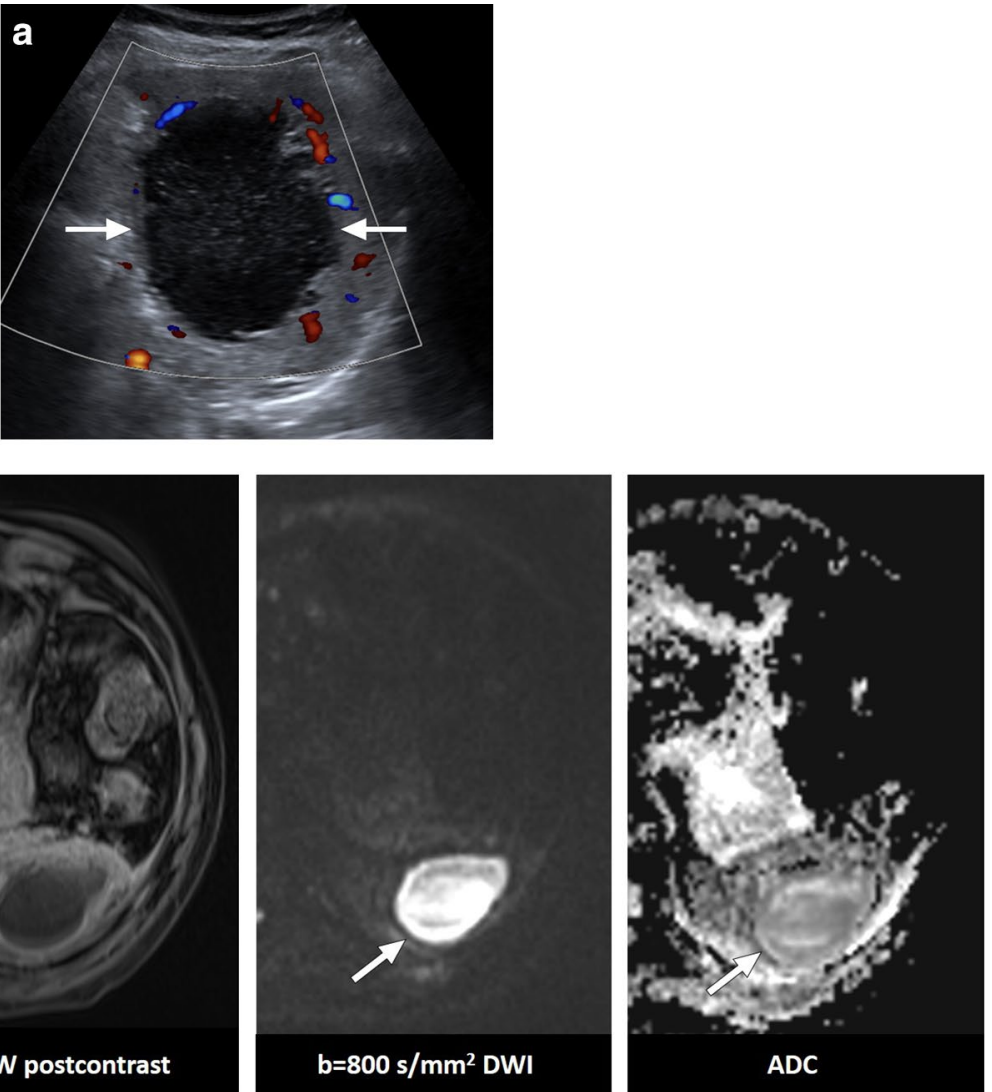

Fig. 7 Splenic abscesses in two different patients: a 31-year-old female with known chronic granulomatous disease presented with left upper quadrant pain and fever. Gray-scale US and color Doppler studies showed a hypoechoic, cystic lesion in the spleen with a thick irregular wall and increased vascularity around the lesion (arrows). Percutaneous drainage revealed pus which grew Gram (+) and Gram $(-)$ bacteria consistent with polymicrobial splenic abscess. The patient's general condition improved shortly after the procedure. b

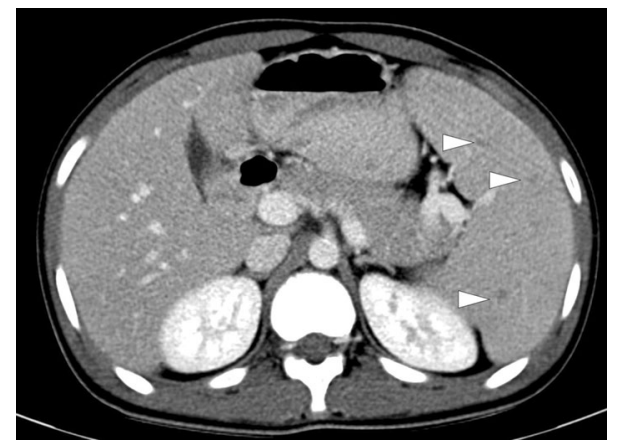

Fig. 8 Splenic brucellosis: 17-year-old male patient with a history of unpasteurized dairy product consumption presented to ER with malaise, prolonged recurrent fever, and dull abdominal pain. Laboratory workup showed anemia and leukopenia with an increase in acute phase reactants. Axial plane postcontrast abdominal CT showed multiple subcentimeter hypodense nodules (arrowheads) in the spleen. With these clinical, radiological, and laboratory findings, brucellosis was considered as putative diagnosis. Subsequently, blood culture grew Brucella spp., and the patient was treated accordingly 63-year-old male with known advanced stage metastatic prostate cancer presented with left upper quadrant pain, fever, chills, and altered mental status. Abdominal MRI demonstrated T2-hyperintense cystic lesion with peripheral contrast enhancement and restricted diffusion (arrows). US-guided percutaneous drainage revealed purulent content, which grew Staphylococcus aureus. The patient's general condition improved immediately after the procedure and the start of antibiotic therapy

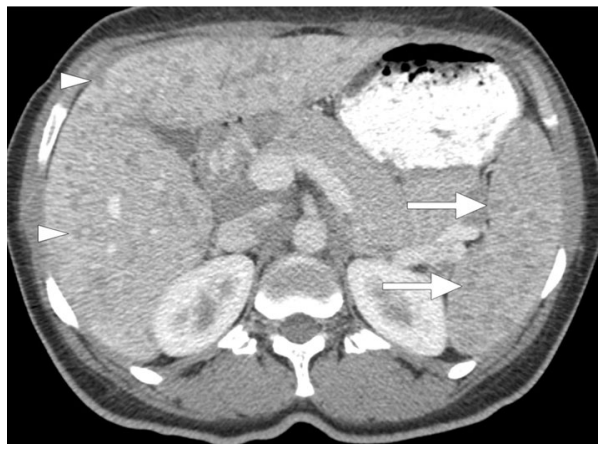

Fig. 9 Splenic tuberculosis: 49-year-old female with known poorly controlled HIV infection presented with fever, weight loss, and persistent cough. Chest CT examination (not shown) and laboratory tests, including sputum specimen, confirmed pulmonary tuberculosis. Axial plane postcontrast abdominal CT image shows the miliary pattern, innumerable sub-centimeter hypodense lesions within the splenic parenchyma (arrows). Also noted were several similar lesions within the liver (arrowheads). Percutaneous US-guided aspiration from the splenic lesions confirmed tuberculosis 
difficult, and most patients require histopathological diagnosis (Fig. 10). The solitary lesions typically appear as hypoechoic focal lesions of variable size on US. With CT, these lesions appear as typically hypodense lesions with heterogeneous internal enhancement. Central necrosis is a common, but again a non-specific, feature of these lesions. Lymphoma is one of the most common initial preliminary diagnoses in these patients as it is a prevalent disease with similar clinical symptoms. In addition to lymphoma, primary aggressive splenic tumors such as angiosarcoma may be considered in the differential diagnosis. Splenic metastases, rare compared to liver metastases, may also mimic mass-forming tuberculomas. The presence of a contrast-enhancing peripheral rind is more suggestive for metastases. Percutaneous imageguided spleen biopsy or splenectomy are the most common approaches for confirming the diagnosis.

\section{Melioidosis}

Melioidosis is caused by a gram-negative bacteria, Burkholderia pseudomallei, which was first described in Burma in 1911 [28]. The primary mode of transmission is contact with contaminated soil or water. It is a systemic disease with high case fatality, between $19 \%$ and $36 \%$, in certain endemic areas [29]. Diabetes mellitus is a significant risk factor that increases the predisposition to the disease and causes the disease to progress more severely [30]. Fever, septicemia, or localized abscesses are common symptoms of the disease, and almost every organ system may be affected, including the spleen.

Spleen is the most commonly involved extrapulmonary organ in the course of the disease. Splenic abscesses may range from 0.5 to $1.5 \mathrm{~cm}$ in size. The lesions may be single or multiple multiloculated lesions, and perisplenic extension may be seen (Fig. 11) [31]. The presence of concurrent liver abscesses in someone who recently traveled from an

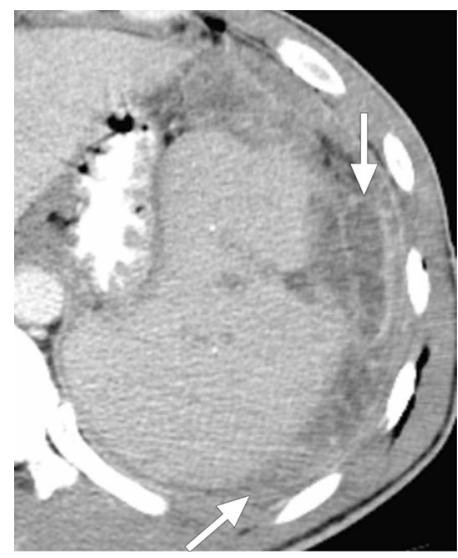

Fig. 11 Melioidosis: 43-year-old male refugee from Southeast Asia presented with fever, fatigue, and left upper quadrant pain. Axial plane postcontrast CT image showed enlarged spleen with predominantly perisplenic collections (arrows). Imaging findings and clinical evaluation were found to be consistent with melioidosis. The patient clinically responded well to the treatment

endemic area in Southeast Asia or northern Australia should raise the clinical suspicion.

\section{Cat scratch disease}

Cat scratch disease (CSD) is a bacterial infection caused by Bartonella henselae. The clinical presentation may be variable depending on the patient's immune status. CSD is mainly seen in immunocompetent hosts such as children and young adults with cat exposure, who present with fever and local lymphadenopathy. Bacillar angiomatosis (BA) is the form of the disease when infection happens in immunocompromised adults such as patients with AIDS [32]. The disease's clinical course is more grave in patients with BA, and disseminated systemic infection may be visible in the liver and spleen [33].

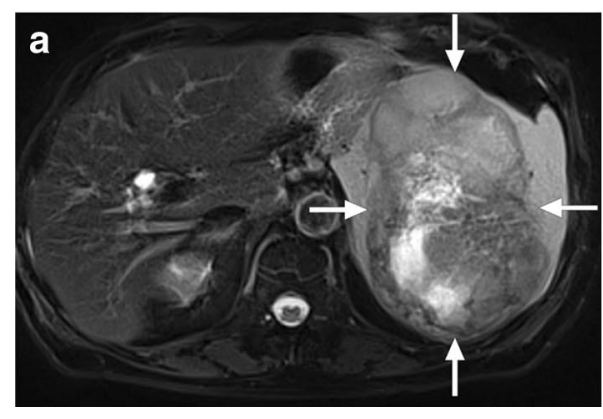

Fig. 10 Tuberculoma: 65-year-old male with no known significant past medical history presented with palpable left upper quadrant mass. a Axial plane T2W image showed a semisolid mass with heterogeneous signal intensity (arrows). b Axial plane postcontrast T1W

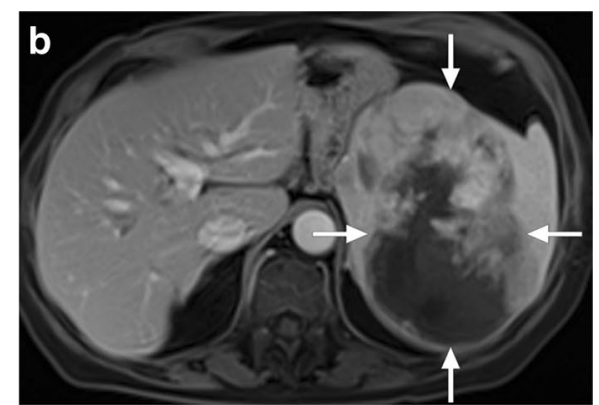

image demonstrated heterogeneous enhancement within the lesion (arrows). As findings were found to be concerning for an angiosarcoma, splenectomy was performed. The final pathologic examination confirmed a giant tuberculoma 
Imaging findings of splenic involvement are mostly related to granuloma formation. The imaging findings are not specific, and lesions typically appear as hypoechoic focal splenic lesions on US [34]. CT is the primary imaging modality in the abdomen, and the lesions typically appear hypoattenuating relative to the background splenic parenchyma (Fig. 12). However, progressive enhancement or rim enhancement has also been observed [35]. On MRI, the splenic lesions appear hypointense on T1W images but moderately hyperintense on $\mathrm{T} 2 \mathrm{~W}$ images [34].

The imaging findings are not specific and may closely resemble splenic involvement in sarcoidosis, candidiasis, or advanced stage metastatic disease. Therefore, obtaining detailed clinical history is mandatory for diagnosis, particularly because confirmatory serology takes time. However, the disease is usually self-limited when the immune system is intact.

\section{Parasitic diseases}

\section{Malaria}

Malaria is among the most common parasitic diseases and represents a major public health problem in endemic parts of the world. Around 219 million cases of this mosquito-borne illness were reported worldwide by World Health Organization in 2018 [36]. Plasmodium falciparum, $P$. vivax, $P$. ovale, $P$. malariae, and $P$. knowlesi have all been implicated but tend to have different geographic distributions [37]. The spleen plays a pivotal role in the course of the disease as the protozoa infect red blood cells, and the spleen is one of the main components of the immune system. Splenomegaly is extremely common.

Splenic infarction or spontaneous rupture during the disease are rare but have been described in the literature as case reports [38]. Cellular hyperplasia and sinusoidal congestion, vascular occlusion leading to thrombotic or ischemic events, and episodic increase in intraabdominal pressure associated with sneezing, coughing, and laughing add additional stress to the already fragile spleen. They have all been mentioned in the splenic rupture and infarction etiopathogenesis in these patients [39]. Additionally, minor and major abdominal trauma may lead to increased abdominal pressure and result in splenic rupture. Splenic rupture appears to be more common in the acute phase of the disease, and this has been attributed mainly to the gradual increase in splenic size in the chronic phase of the disease [39, 40]. Clinically, acute onset pain in the left upper quadrant is typical. Hypotension may also be seen in patients with spontaneous splenic rupture when the bleeding is profuse.

In patients with spontaneous rupture, subcapsular hematoma with associated peri-splenic blood products is characteristic for diagnosis [41]. In endemic areas, repeated subclinical bleeding followed by healing can lead the spleen to develop a so-called "onion skin" appearance [42].

\section{Splenic hydatid disease}

Hydatid disease is a major health problem in certain parts of the world. Virtually any organ may be involved in the course of the disease, where the liver and lungs are the most commonly affected organs. Splenic involvement, especially isolated involvement, is extremely rare and typically occurs in association with disease in the liver. The prevalence of splenic involvement in hydatid disease in the medical literature is between 0.9 and $8 \%$ [43].

On US, the detection of daughter cysts in the lesion is very typical for the diagnosis, but the content of the lesion may vary highly depending on the stage of the disease [43, 44].

On CT and MR, the absence of contrast enhancement, both in the septae and the wall, is typical. On CT, these

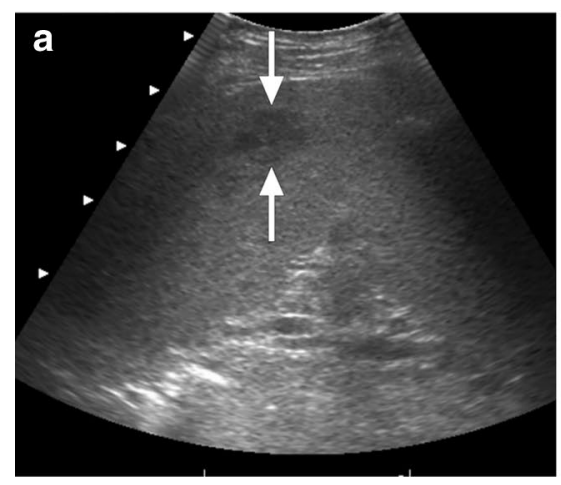

Fig. 12 Cat scratch disease: 16-year-old female with no known past medical history presented with swelling in her right hand, fever, and enlarged spleen on physical exam after being scratched by her domestic cat. a US image showed an enlarged spleen with a small hypo-

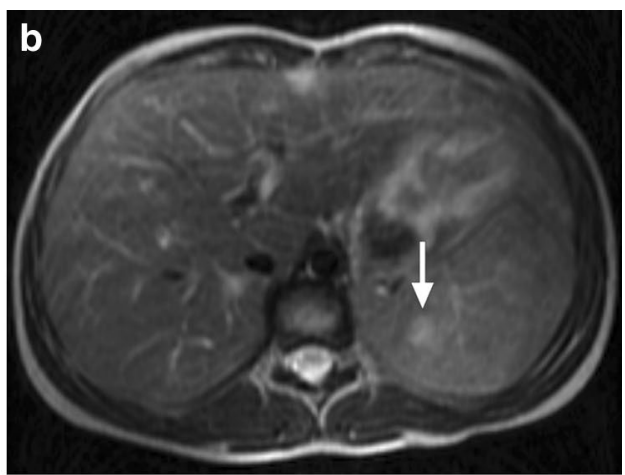

echoic solid lesion within the parenchyma (arrows). b Axial plane T2W image demonstrated the same lesion as hyperintense focus (arrow). Serologic studies confirmed cat scratch disease, and the lesion disappeared on follow-up imaging 
lesions appear as hypoattenuating lesions. Daughter cysts, when present, may appear as even lower attenuation circular lesions within the cyst. T2W MR images are more helpful for outlining the internal structure of the cyst (Fig. 13). The imaging characteristics may generally allow correct diagnosis.

Secondary infection, rupture into the peritoneal cavity and fistulization to the adjacent viscera are among the major complications of splenic hydatid disease. Rupture can be seen spontaneously as well as secondary to trauma and may result in a life-threatening anaphylactic reaction (Fig. 14) [45]. Surgery and percutaneous treatments may both be used for the treatment, and the therapeutic approach mainly

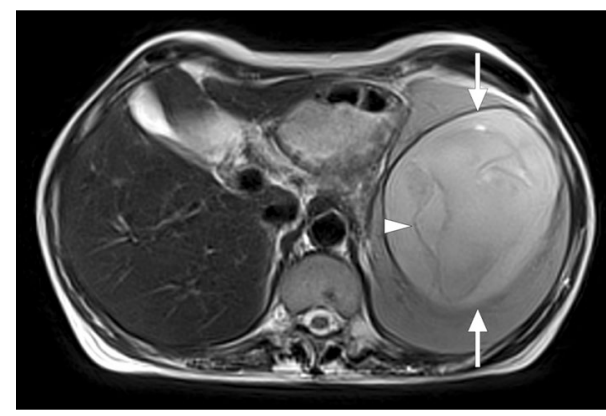

Fig. 13 Splenic hydatid disease: 47-year-old female with no significant past medical history presented with left upper quadrant pain and splenomegaly. Axial plane T2W abdominal MR image shows a wellcircumscribed distended cystic lesion (arrows) with associated inner membranes (arrowhead) consistent with hydatid cyst. The patient underwent percutaneous treatment depends on the disease stage and local expertise [44]. In the current series, it is emphasized that percutaneous treatment is a safe and reliable approach, and anaphylaxis has not been reported [46, 47]. However, the possibility of an anaphylactic reaction after splenic puncture should be considered during the procedure, and caution should be exercised.

\section{Leishmaniasis}

Leishmaniasis is a chronic zoonotic disease transmitted by an obligate intracellular protozoan transmitted by sandfly bites [48]. In visceral leishmaniasis, a chronic, insidious form of the disease, the parasites may migrate into several internal organs such as the liver, spleen, and bone marrow. The diagnosis of this disease may be challenging, and the prognosis may be dismal in untreated patients.

There is not much information on the imaging findings of splenic involvement in the literature, and only anecdotal case reports have been published [49]. The typical imaging appearance is multiple lesions within the splenic parenchyma which may appear hypodense on CT and hypointense on T2W images on MR images (Fig. 15). The T2W hypointensity on MR may help differentiate these lesions from other diseases such as splenic metastases. Rim-like enhancement with central hypo-enhancement was reported in splenic leishmaniasis. The absence of restricted diffusion on MRI may also be a helpful imaging clue [49]. Sarcoidosis and granulomatous infections of the spleen may have similar imaging findings with leishmaniasis and should also be considered in the differential diagnosis.
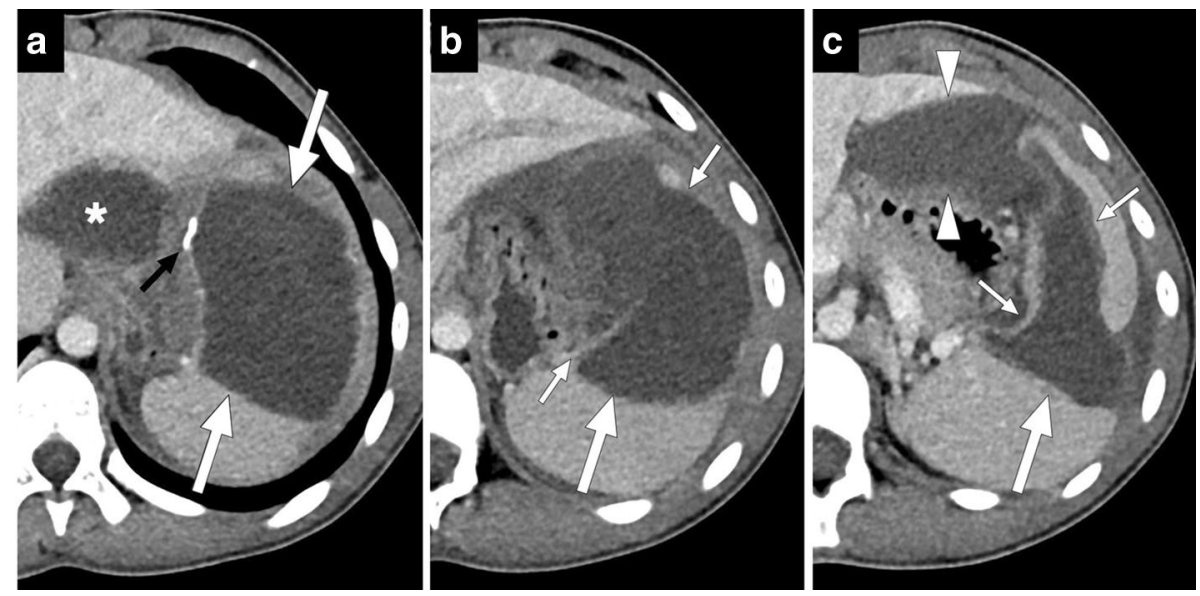

Fig. 14 Ruptured splenic hydatid cyst: 15-year-old male presented to ER with sudden onset abdominal pain, diffuse skin rash, dyspnea, and hypotension. After stabilization, thoracoabdominal CT was obtained for further investigation. a-c Consecutive postcontrast abdominal CT slices from superior to inferior showed a large cystic lesion (large white arrows) with a partially calcified wall (small black arrow) in the spleen. a Note was also made of another cystic lesion in the left liver lobe (asterisk). b There was a wall discontinuity in the inferomedial part of the splenic lesion (small white arrows). $\mathbf{c}$ In the most inferior slices, the contours of the lesion had a wavy appearance, suggestive of the loss of internal pressure (small white arrows). Extrasplenic extension of the hypodense lesion content (arrowheads) and mild perisplenic free fluid were also noted. With this clinical presentation and radiological findings, anaphylaxis secondary to the splenic hydatid cyst rupture was considered as putative diagnosis. An urgent surgery confirmed the diagnosis 


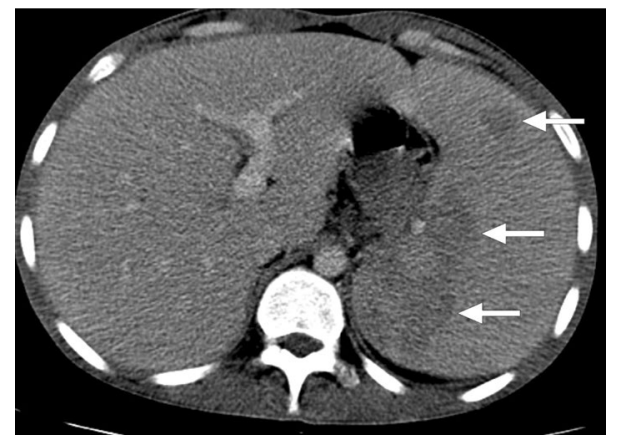

Fig. 15 Leishmaniasis: 25-year-old male patient with a history of unexplained fever, pancytopenia, and increased serum C-reactive protein levels was found to have splenomegaly and parenchymal heterogeneity on US image (not shown). Axial plane postcontrast CT image showed severely enlarged spleen and large hypodense areas (arrows). Histopathologic examination after splenectomy confirmed leishmaniasis

\section{Babesiosis}

Babesiosis is a tick-borne parasitic infectious disease caused by the protozoa of the genus Babesia. Once mostly limited to New England's coastal areas, it is now seen throughout the whole USA [50]. Lyme disease has a similar distribution, relating to the common tick vector-Ixodes scapularis. The disease tends to be particularly severe in asplenic individuals. In patients with their spleen but sick enough to require hospitalization, splenic abnormalities will often be found, with splenomegaly as the most common imaging finding [51].

Splenomegaly seems to be the most common imaging finding, but splenic infarcts and ruptures have also been reported (Fig. 16) [51]. The underlying pathogenesis of splenic rupture has been proposed to be the degradation and friability of the parenchyma and not the enlargement and elevated intra-capsular pressure [52]. Middle-aged healthy men with intact immune system were reported to be the patient group with the highest risk for splenic rupture [53, 54].

\section{Opportunistic infections}

\section{Candidiasis}

Splenic fungal infections, candidiasis being the most common, is primarily the disease of immunocompromised patients. Concurrent infection in the liver is very common in patients with splenic candidiasis. Focal embolic parenchymal abscesses appear as sub-centimeter hypodense lesions on CT (Fig. 17). The hypoattenuating nature of these lesions is due to necrosis. Associated parenchymal infarcts may also be observed in the course of the disease.

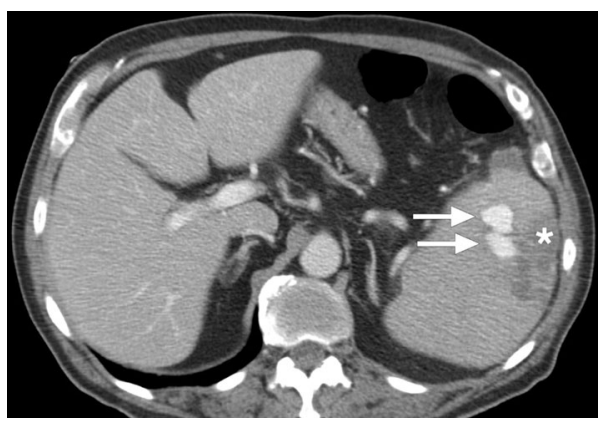

Fig. 16 Babesiosis: 83-year-old male with acute babesiosis. Axial plane postcontrast abdominal CT image shows splenomegaly, splenic infarcts (asterisk), and splenic pseudoaneurysms (arrows). The patient was subsequently treated with endovascular embolization of the pseudoaneurysm (not shown)

\section{Nocardiosis}

Nocardia is weakly acid-fast, aerobic, gram-positive, branching, filamentous bacteria. It may cause localized or systemic suppurative infections. Systemic nocardiosis is primarily seen in patients with immune suppression, particularly those with cell-mediated immunity problems [55]. Despite the lungs being the most common infection site, the organism may spread almost anywhere in the body from a pulmonary or cutaneous source [56]. Hypodense, non-enhancing sub-centimeter nodules within the splenic parenchyma appear to be the most common imaging finding (Fig. 18). In the presence of known cutaneous or pulmonary infections, the diagnosis may be easier.

\section{Mycobacterium avium-intracellulare complex}

In the course of systemic mycobacterial infections, the spleen is a commonly involved organ [57]. Disseminated Mycobacterium avium-intracellulare (MAC) infection is particularly common in HIV/AIDS patients with low CD4 counts [58]. Despite this high rate of splenic infection, imaging findings have not been reported in detail [57]. The imaging findings may be similar to splenic TB and mostly are visualized as non-specific small hypodense structures on CT. On MRI, they appear to have low signal intensity on $\mathrm{T} 1 \mathrm{~W}$ in-phase imaging and DWI due to the ferromagnetic contents. T2-hypointensity and progressive peripheral contrast enhancement are other considerable imaging findings (Fig. 19) [58].

Pulmonary involvement is relatively common, and the main imaging findings are bronchiectasis, bronchial wall thickening, mucus plugs, small centrilobular nodules, treein-bud appearance, airspace consolidation, and cavities [59]. The presence of accompanying pulmonary involvement can 

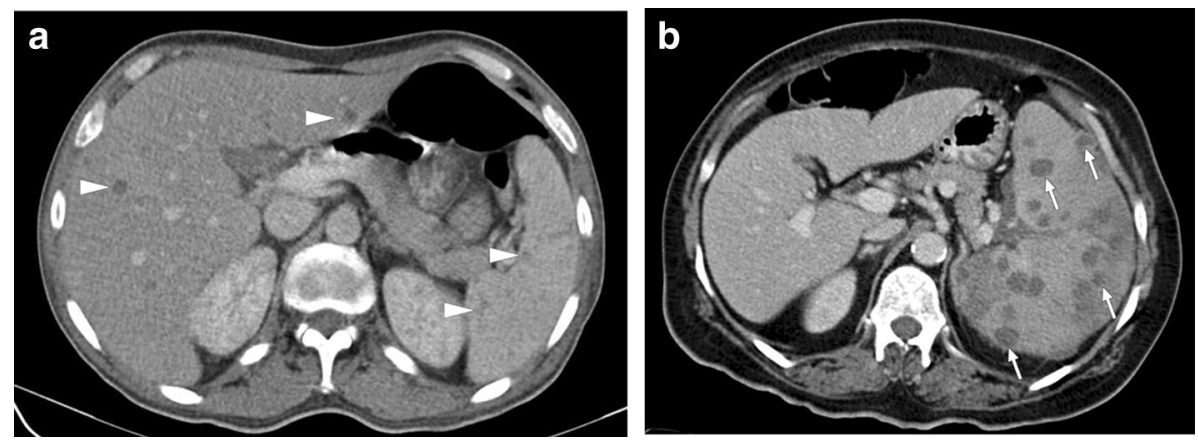

Fig. 17 Candidiasis in two different patients: a 43-year-old female with acute myeloid leukemia who had been undergoing chemotherapy acutely developed fever and abdominal pain. Axial plane postcontrast abdominal CT image showed multiple sub-centimeter hypodense lesions scattered throughout the liver and the spleen parenchyma (arrowheads). Imaging findings were found to be consistent with opportunistic candida infection. The patient clinically responded well, and the lesions almost completely disappeared on follow-up (not shown). b 62-year-old female with known acute

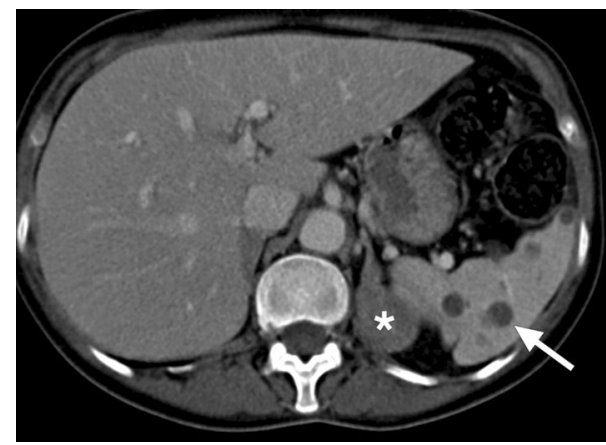

Fig. 18 Nocardiosis: 57-year-old female who was on high dose steroid for severe nephrotic syndrome presented with fever and abdominal pain. Axial plane postcontrast abdominal CT image showed welldefined hypodense lesions scattered through the splenic parenchyma (arrow). Also noted was a left adrenal mass (asterisk) which was later confirmed to be a large adenoma after laparoscopic resection. Percutaneous US-guided aspiration of splenic cysts revealed Gram-positive branched rods consistent with nocardiosis

make this diagnosis more likely to be considered and may facilitate the differential diagnosis.

\section{Pneumocystosis}

Pneumocystis jirovecii is the causative agent of pneumocystosis, which had been a rare infection before the HIV/AIDS pandemic, primarily observed in patients with immunosuppression related to pharmacotherapy $[60,61]$. Pneumonia is the most common presentation of this infection, but its disseminated form may involve the spleen, liver, gastrointestinal tract, heart, bone marrow, kidneys, thyroid, and skeletal muscle. In patients with inconclusive clinical findings, lymphoblastic leukemia presented with neutropenic fever. Axial plane postcontrast abdominal CT showed splenomegaly with heterogeneous parenchyma and multiple hypoattenuating, well-defined nodular lesions within the spleen (arrows). Systemic candidiasis was considered as the leading diagnosis, and IV antifungal treatment was immediately started after obtaining blood culture. The patient improved within a few days. Blood culture grew Candida spp. the day after the CT study

diagnosis may be established with fine-needle aspiration biopsy [62].

On CT, splenic foci appear as hypodense lesions of variable size scattered throughout the splenic parenchyma. However, large size, solid-appearing lesions may also be observed (Fig. 20) [62].

\section{Actinomycosis}

Actinomycosis is a chronic granulomatous condition which is caused by Actinomyces species. The cervicofacial region is the most common anatomic compartment affected by systemic infection. However, abdominopelvic involvement may be seen in around 20\% of the cases [63]. Immunosuppression is a well-known predisposing risk factor for systemic infection. Splenic abscesses appear to be extremely rare but have been reported in the literature as anecdotal case reports [64]. On imaging, the abscesses appear as cystic lesions with variable internal echotexture a honeycomb appearance (Fig. 21) [64].

\section{Rare fungal infections of the spleen}

Coccidioides immitis is a dimorphic fungus, endemic in the southwestern United States [65]. The disease's symptomatology may range from asymptomatic disease to disseminated fatal disease. The disease is usually acquired from dust exposure from the soil containing the infectious spores. HIV infection and other immunosuppressive conditions may predispose patients to disseminated disease [66]. Splenic involvement is rare and was reported in the disseminated form of the disease. Multiple granulomas caused by the 

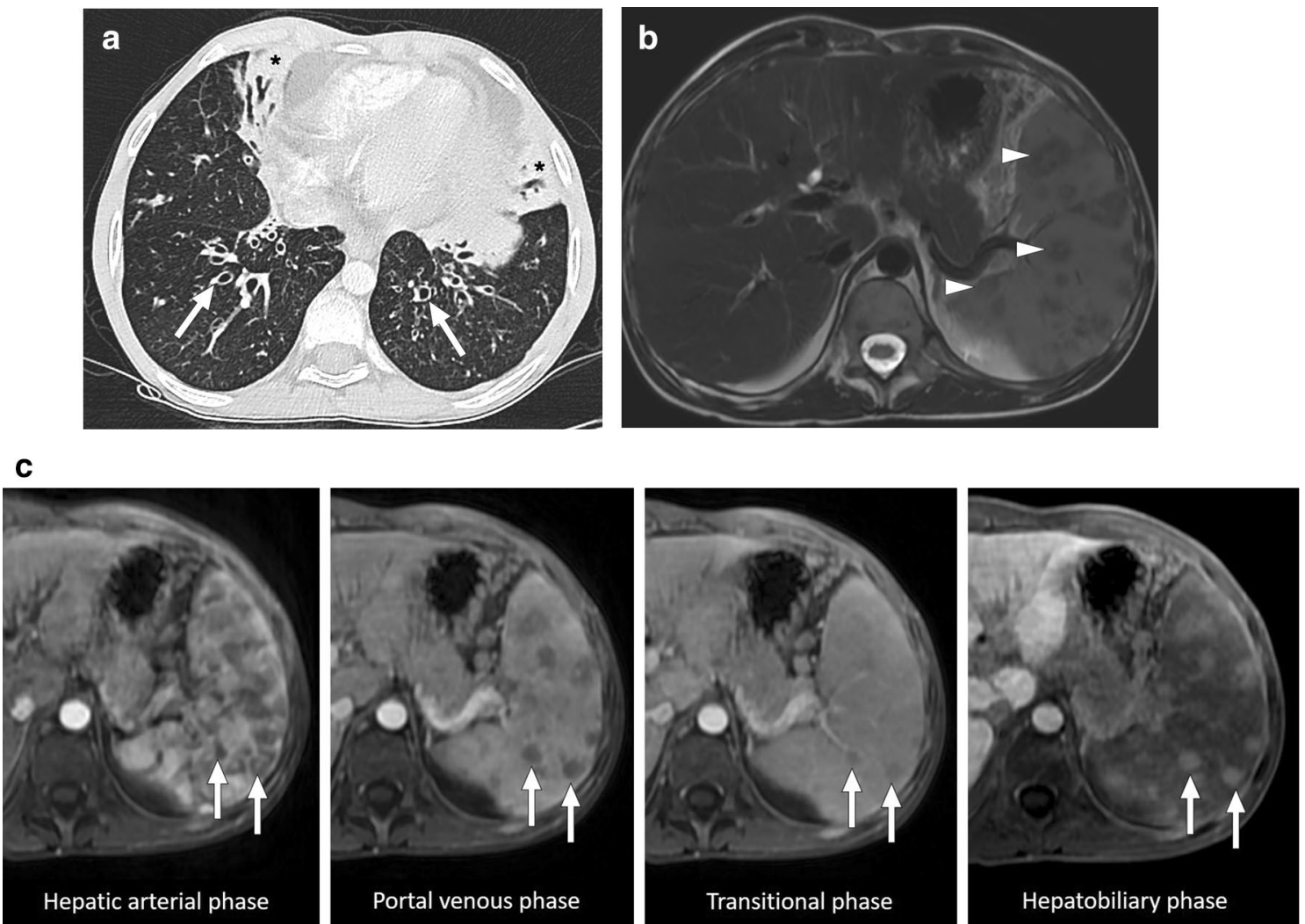

Fig. 19 Mycobacterium avium-intracellulare infection: 17-year-old male patient with a known congenital immunodeficiency syndrome presented to ER with complaints of fever, malaise, chest pain, and left upper quadrant abdominal pain. a Chest CT showed bronchiectasis (arrows), mucus plugs, and centrilobular nodules in the lower lobes. Note was also made of airspace consolidation and volume loss in the right middle lobe and lingula (asterisks). Imaging findings were found to be suggestive of pulmonary atypical mycobacterial infection. b Axial plane T2W abdominal MR image of the same patient demonstrated multiple nodular hypointense lesions of varying sizes within the spleen (arrowheads). c In dynamic postcontrast MRI images with

hepatospecific contrast agent, these lesions were hypoenhancing in the early phases. However, the signal intensity difference between the lesions and background splenic parenchyma gradually decreased in later phases (arrows). The lesions were more hyperintense than the splenic parenchyma in the hepatobiliary phase (arrows), possibly due to their fibrotic nature causing delayed contrast retention. With these imaging findings, splenic granulomas due to the atypical mycobacterial infection were considered. Sputum culture and PCR confirmed Mycobacterium avium-intracellulare complex infection, and the patient was treated accordingly

Fig. 20 Pneumocystosis: An elderly female patient presented with incidentally detected splenic lesions. a Coronal plane T2W MR image showed hypointense lesions in the spleen (arrows) b Coronal plane postcontrast T1W image demonstrated peripheral enhancement in both lesions (arrows). Image-guided aspiration confirmed Pneumocystis jirovecii
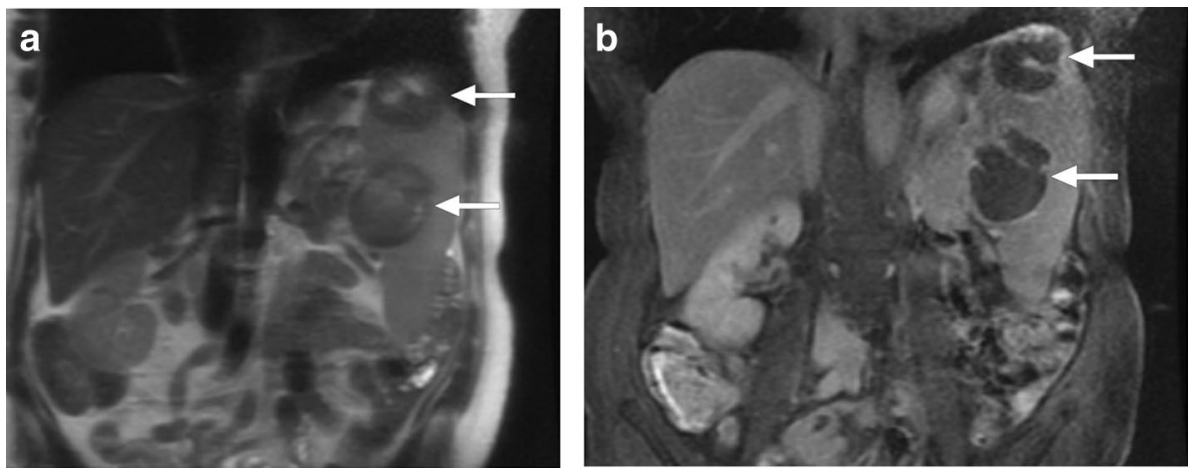

fungi are typically seen as sub-centimeter hypodense nodules within the splenic parenchyma [67].

Histoplasma capsulatum is an endemic mycosis and a common infection source in North America, South America,
Africa, and Asia. The main route of transmission is via the inhalation of spores from contaminated soil [68]. Lungs are common sites for infection, but disseminated infection may be observed in immunosuppressed individuals. Symptomatic 


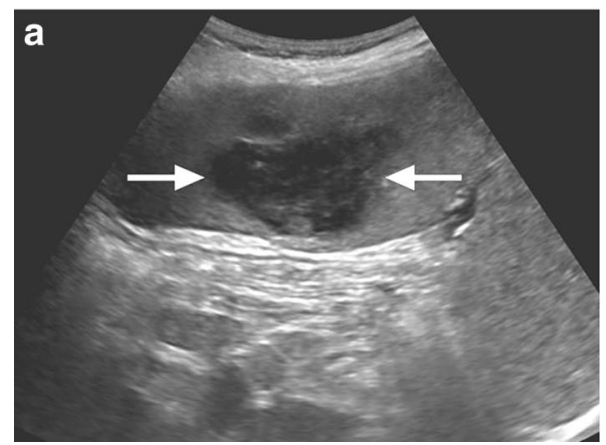

Fig. 21 Actinomycosis in two different patients: a 24-year-old female with a history of chronic granulomatous disease presented with fever and left upper quadrant pain. Her spleen was palpable on physical examination. Gray-scale US exam demonstrated a cystic lesion with septations in the upper pole of the spleen (arrows), which was confirmed to be consistent with an abscess due to Actinomyces israelii.

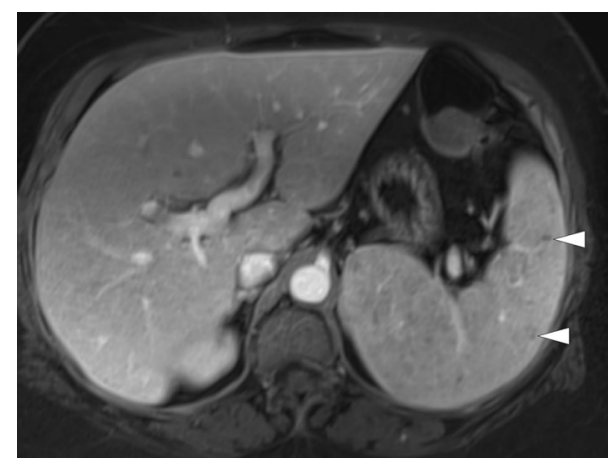

Fig. 22 Histoplasmosis: 62-year-old female patient receiving methotrexate and TNF-alpha antagonist for treatment-resistant rheumatoid arthritis presented with fever and left upper quadrant pain. Axial plane T2W-fat-suppressed MR image showed several subcentimeter hypointense lesions scattered throughout the spleen (not shown). These lesions did not demonstrate any obvious enhancement in axial plane postcontrast T1W image (arrowheads). Percutaneous imageguided biopsy revealed non-necrotizing granulomas associated with budding yeast consistent with Histoplasma capsulatum infection

disease is rare in immunocompetent hosts. Splenic involvement is typically seen as small hypoenhancing lesions on CT and MR, similar to other infectious diseases involving the spleen (Fig. 22). More commonly, calcified granulomas are incidental findings secondary to previous asymptomatic infection in patients from endemic areas such as the Ohio and Mississippi River Valleys of the northeastern USA [69].

Cryptococcus neoformans is a cause of infection mostly in immunocompromised patients, particularly in HIVinfected cases. It is commonly found in soil contaminated by bird droppings and human infection is most commonly by inhalation of the yeast spores. Pulmonary infection is common, but splenic microabscesses are rare [70]. On imaging, these microabscesses are similar to other fungal splenic

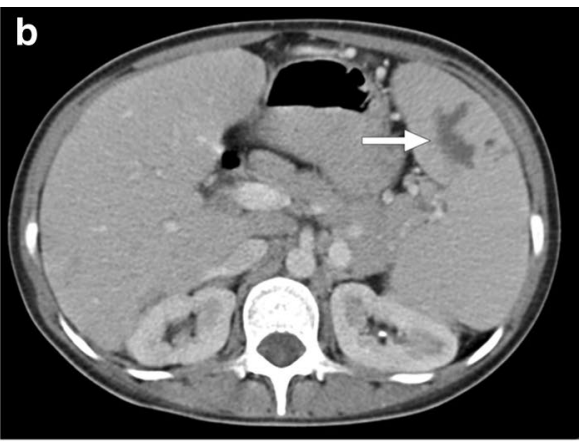

b 30-year-old female patient with acute myeloid leukemia presented with intermittent low-grade fever and abdominal discomfort. Axial plane postcontrast abdominal CT showed hypodense branching lesions (arrow). US-guided aspiration and culture grew gram-positive filamentous branching bacilli consistent with Actinomyces spp

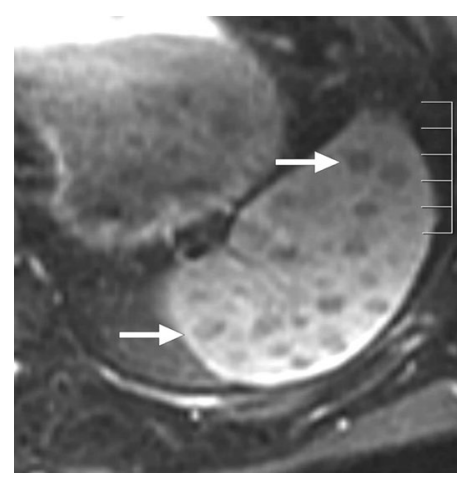

Fig. 23 Cryptococcosis: 35-year-old male patient with poorly-controlled HIV infection presented with high-grade fever and severe fatigue. Abdominal US examination demonstrated several hypoechoic lesions within the spleen (not shown). Axial plane postcontrast T1W MR image showed innumerable hypoenhancing sub-centimeter lesions within the splenic parenchyma (arrows). Percutaneous imageguided biopsy from the spleen confirmed cryptococcal infection

involvements. They appear as low attenuation lesions typically between $5 \mathrm{~mm}-10 \mathrm{~mm}$ in diameter with a central area of hypoattenuation (Fig. 23) [70]. However, as imaging characteristics are similar to other fungal opportunistic infections, biopsy confirmation from the spleen or other involved organs is almost always necessary.

Invasive aspergillosis is an important cause of morbidity and mortality, especially in immunosuppressed patients. The primary infection source is mostly the lungs, and hepatosplenic involvement has been reported in up to $15 \%$ of the patients. Disseminated disease has also been reported in immunocompetent patients, although very rarely [71]. On $\mathrm{CT}$, splenic involvement has been mostly reported to be seen as multiple hypoattenuating lesions within the splenic parenchyma (Fig. 24). In rare cases, pseudoaneurysms and parenchymal infarcts were also detected [72]. Radiological 


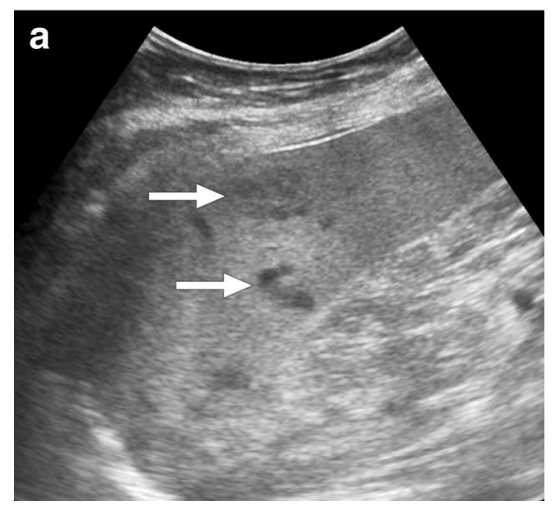

Fig. 24 Aspergillosis: 59-year-old female patient with a history of chemoradiotherapy for head/neck cancer presented with fever, malaise, and weight loss. a Longitudinal plane gray-scale abdominal US image showed multiple hypoechoic nodular lesions within the spleen (arrows). b Axial plane postcontrast abdominal CT image

findings are not specific, and the presence of branching septate fungal hyphae in the pathological specimen is diagnostic.

\section{Involvement patterns in splenic infectious diseases with differential diagnoses}

Basically, there are four fundamental imaging patterns of splenic involvement in the course of infectious diseases: (1) Splenomegaly without focal lesion, (2) multinodular pattern (representing micro-abscesses), (3) predominantly cystic lesion (larger abscesses), and rarely (4) mass-forming solid parenchymal lesions [1]. Splenic infarcts and splenic rupture are relatively rare findings that may develop during some infections. As can be seen in many other diseases, splenic calcifications may also appear, especially in the later stages of infection. These imaging features may be seen in isolation or together in the same patient. A summary of differential diagnosis of splenic diseases based on these fundamental imaging patterns can be found in Table 2 .

\section{Splenomegaly without focal lesion}

As a non-specific imaging finding, splenomegaly can be seen due to many different entities [73]. Infectious mononucleosis is one of the most common infectious causes of splenomegaly. In addition to Epstein-Barr virus (EBV), cytomegalovirus (CMV), human immunodeficiency virus (HIV), and toxoplasmosis may also cause mononucleosis-like syndrome and present with diffuse splenomegaly without focal parenchymal involvement [74]. Infective endocarditis, typhoid fever, brucellosis, and second-stage syphilis may be counted among the other less common causes of diffuse

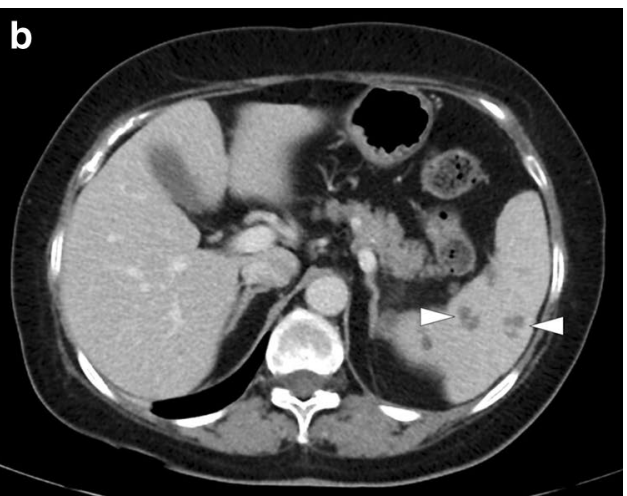

demonstrated the same lesions as multiple hypoattenuating nodules (arrowheads). US-guided percutaneous biopsy was performed, and histopathological examination revealed branching septate fungal hyphae. Tissue culture grew Aspergillus spp

splenic enlargement. In addition to these bacterial infections, well-known parasitic diseases such as malaria and leishmaniasis may present with massive splenomegaly [75].

In addition to infectious causes, several other non-infectious etiologies such as congestive heart failure, portal hypertension, lympho/myeloproliferative, and rheumatological diseases may also cause splenomegaly [73, 75]. Imaging alone is mostly not sufficient for the diagnosis of the etiology of splenomegaly. A comprehensive clinical evaluation with extensive laboratory and imaging workup is also mandatory.

\section{Multinodular pattern}

This pattern mainly represents multiple parenchymal microabscesses and granulomas. Splenic micro-abscesses are mostly seen in the course of opportunistic fungal infections. They are typically seen as multiple small (usually less than 5-10 $\mathrm{mm}$ in diameter) lesions scattered throughout the splenic parenchyma. Granulomas may appear as hypoenhancing nodules at earlier phases; however, they may show gradual contrast retention at later phases [76, 77].

Tuberculosis, nocardiosis, and cat-scratch disease are the typical bacterial infections that may present with the multinodular pattern in the spleen. Parasitic diseases such as leishmaniasis may also give rise to parenchymal nodules in an enlarged spleen [78].

Among the non-infectious causes of multinodular pattern sarcoidosis, lymphoproliferative and rheumatologic diseases, hemangiomatosis, lymphangiomatosis, and metastases may be counted $[3,6-8,76]$.

Concomitant liver or lung involvement, hepatosplenomegaly, and enlarged lymph nodes in the abdomen may be helpful clues for diagnosing sarcoidosis (Fig. 25). The low signal intensity of the parenchymal nodules in all MRI 
Table 2 Imaging features of splenic diseases with differential diagnosis

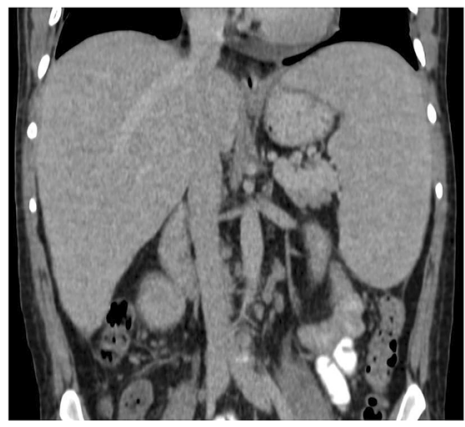

Non-mass splenomegaly:

$>$ Infectious

* Mononucleosis, CMV, HIV

* Infective endocarditis

* Typhoid fever,brucellosis

* Syphilis, leptospirosis

* Malaria, Leishmaniasis, Babesiosis

\section{$>$ Non-infectious}

* Portal hypertension and cirrhosis

* Congestive heart failure

* Lympho/myeloproliferative diseases

* Hematologic diseases

* Rheumatologic/inflammatory diseases

* Metabolic and storage diseases

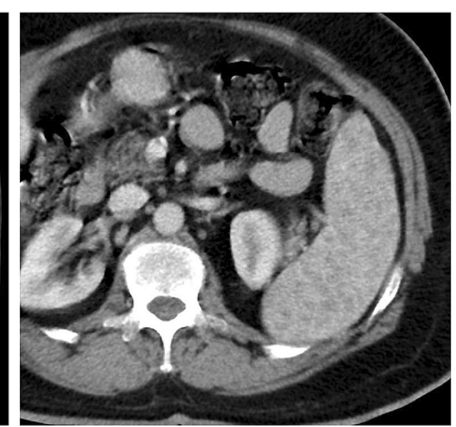

Multiple nodular lesions:

$>$ Infectious

* Tuberculosis/MAC,melioidosis, brucellosis

* Cat scratch disease, nocardiosis

* Fungal infections

* Leishmaniasis

\section{$>$ Non-infectious}

* Pitfall (inappropriate imaging phase)

* Gamna-Gandhi nodules

* Lymphoproliferative diseases

* Metastases

* Sarcoidosis

* Hemangiomatosis, Lymphangiomatosis

* Littoral cell angioma, peliosis

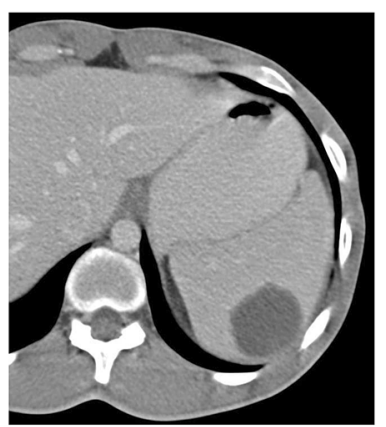

Cystic lesions:

$>$ Infectious

* Pyogenic bacterial abscess

* Melioidosis/Glanders

* Actinomycosis

* Nocardiosis

* Hydatid cyst

\section{$>$ Non-infectious}

* Secondary splenic cyst (due to trauma of infarction)

* Hematoma (chronic phase)

* Epidermoid cyst

* Lymphangioma

* Splenic peliosis

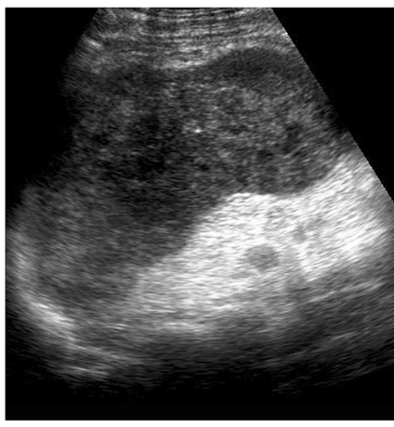

Mass-forming solid lesions:

Infectious (rarely)

* EBV-associated pseudotumor

* Tuberculoma

* Pneumocystosis

\section{$>$ Non-infectious}

* Hematoma

* Lymphoproliferative diseases

* Extramedullary hematopoiesis

* Splenic solid lesions (hamartoma, hemangioma, SANT, angiosarcoma)

* Sarcoidosis

* Metastases

* Gaucher nodules

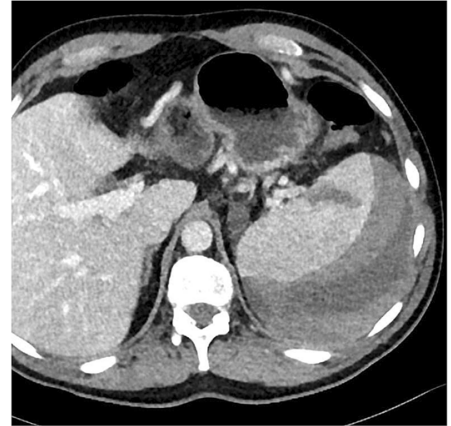

Splenic rupture:

$>$ Infectious

* Mononucleosis, CMV, HIV

* Infective endocarditis, Melioidosis

* Cat scratch disease, Syphilis, Legionellosis

* Malaria, Babesiosis, Leishmaniasis

\section{$>$ Non-infectious}

* Trauma, iatrogenic, local inflammation

* Lympho/myeloproliferative diseases

* Primary splenic diseases ( malignancy, splenic cyst, peliosis, angiomatosis, infarction)

* Portal hypertension

* Rheumatologic diseases

* Hematologic diseases, coagulopathy

* Metabolic and storage diseases

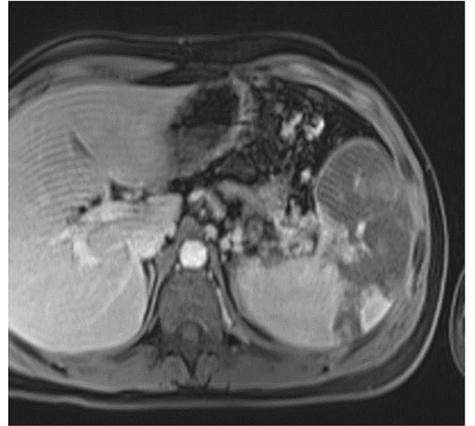

Splenic infarction:

$>$ Infectious

* Mononucleosis, CMV

* Infective endocarditis, melioidosis

* Malaria, Babesiosis, Leishmaniasis

\section{$>$ Non-infectious}

* latrogenic

* Lymphoproliferative diseases

* Malignancies

* Portal hypertension

* Thrombophilia, Vasculitis

* Splenic artery involving diseases

* Sickle cell anemia, Embolic etiologies

* Storage diseases

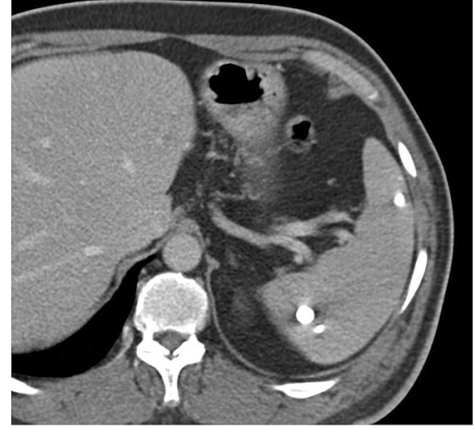

Calcification:

$>$ Infectious

* Fungal infections (candidiasis, pneumocystosis, histoplasmosis)

* Tuberculosis, Brucellosis

* Hydatic cysts (inactive lesions)

\section{$>$ Non-infectious}

* Splenic cysts (wall calcification)

* Hemangioma(tosis), lymphangioma(tosis)

* Hamartoma

* Splenic infarcts

* SLE, amyloidosis

* Sickle cell disease

* Treated Hodgkin lymphoma sequences is a well-known imaging feature of sarcoidosis involvement in the spleen [79].

The imaging and clinical features of abdominal sarcoidosis are very similar to patients with lymphoma. Splenomegaly, multiple small splenic nodules, and conglomerating enlarged lymph nodes are well-known imaging features of lymphoma (Fig. 2). On MRI, T2W signal intensity is mostly not very helpful $[6,80]$.

Lymphangiomatosis, currently known as generalized lymphatic anomaly, is a disease characterized by multiple 


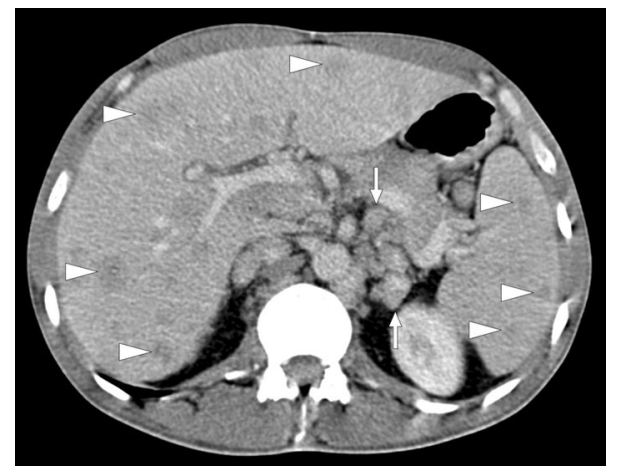

Fig. 25 Histopathologically proven splenic sarcoidosis: 36-year-old male patient with new-onset low-grade fever and cough. Axial plane postcontrast abdominal CT image shows multiple hypodense nodular lesions in both the liver and spleen (arrowheads). Note was also made of multiple enlarged lymph nodes in the paracaval and paraaortic regions (arrows)

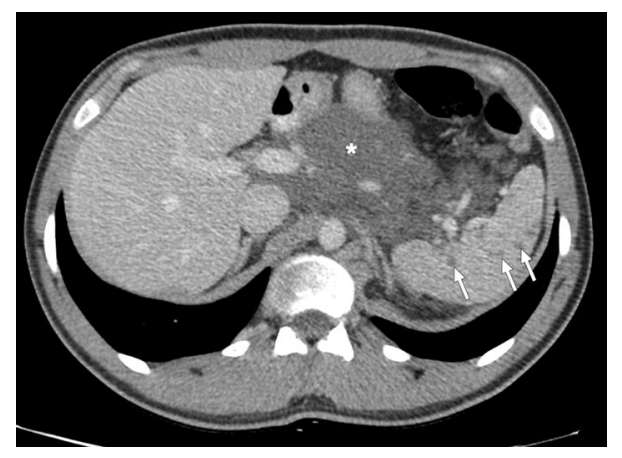

Fig. 26 Splenic lymphangiomatosis: 21-year-old male patient with histopathologically-proven generalized lymphatic anomaly. Axial plane postcontrast CT showed large peripancreatic lymphangioma extending into the gastrohepatic and gastrosplenic ligaments (asterisk) and multiple hypodense subcentimeter nodules representing lymphangiomas scattered throughout the splenic parenchyma (arrows) lymphangiomas with cutaneous, osseous, and visceral involvement and may cause a multinodular pattern in the spleen (Fig. 26) [81, 82]. Hemangiomatosis and littoral cell angioma are vascular neoplastic pathologies that may also present with the multinodular pattern (Fig. 27) [83, 84]. T2W hyperintensity is expected in hemangiomas and lymphangiomas, while littoral cell angiomas can be observed as both $\mathrm{T} 2 \mathrm{~W}$ hyper- or hypointense lesions depending on the iron content [7].

Splenic metastases may also be seen as parenchymal nodules or bulky masses in the spleen (Fig. 28). In the presence of a history of primary malignancy, metastases should also be strongly considered. PET/CT with 18-fluorodeoxyglucose (FDG) can help distinguish metastases from benign lesions, especially in patients with a history of malignancy [66]. Close imaging follow-up to determine the temporal evolution of lesion size or histopathological examination may be considered depending on the patient characteristics [76].

Systemic inflammatory diseases such as hemophagocytic lymphohistiocytosis (Fig. 1) and Still's disease (Fig. 29) may also cause parenchymal nodules and may closely mimic splenic infections or lymphoma both clinically and radiologically. Extensive clinical and laboratory workup is needed for differential diagnosis [20,85].

Despite the fact that they generally do not pose diagnostic difficulty, Gamna-Gandy bodies should be considered in the setting of multiple splenic nodules. A history of recurrent blood transfusions, chronic liver disease, and portal hypertension may facilitate the diagnosis. On US, they appear as small hyperechoic nodules with variable posterior acoustic shadowing depending on the amount of calcification. On MRI, these siderotic parenchymal nodules are the main imaging finding [86]. They appear as hypointense in both $\mathrm{T} 2 \mathrm{~W}$ images and gradient-echo sequences due to the magnetic susceptibility effect of iron. T2*W and

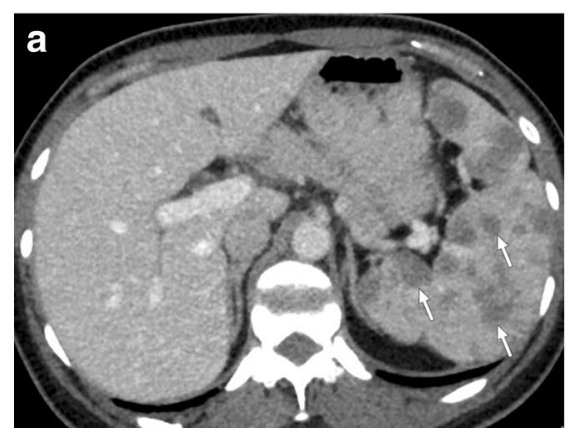

Fig. 27 Histopathologically proven splenic hemangiomatosis in two patients. a 42-year-old female patient with no significant past medical history presented to ER with fever and malaise. Axial plane postcontrast abdominal CT image demonstrated multiple nodular hypodense lesions in the spleen (arrows). Infectious etiologies were considered, and a US-guided percutaneous biopsy was performed. Histopatholog-

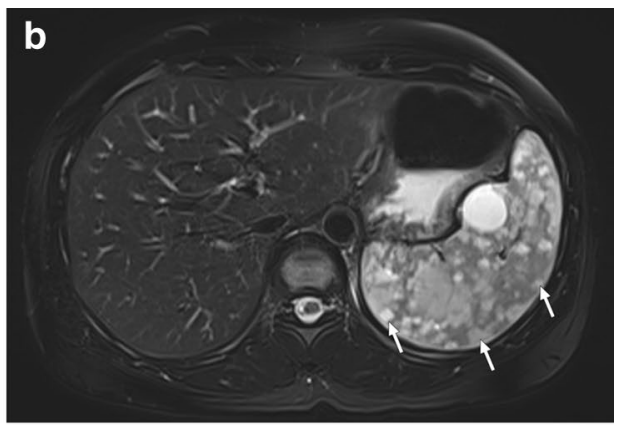

ical examination confirmed splenic hemangiomatosis. b 34-year-old female patient presented with left upper quadrant pain. Axial plane T2W-fat-suppressed abdominal MR image showed multiple hyperintense nodular lesions (arrows) almost completely replacing the splenic parenchyma. Splenectomy was performed, and histopathological examination confirmed splenic hemangiomatosis 


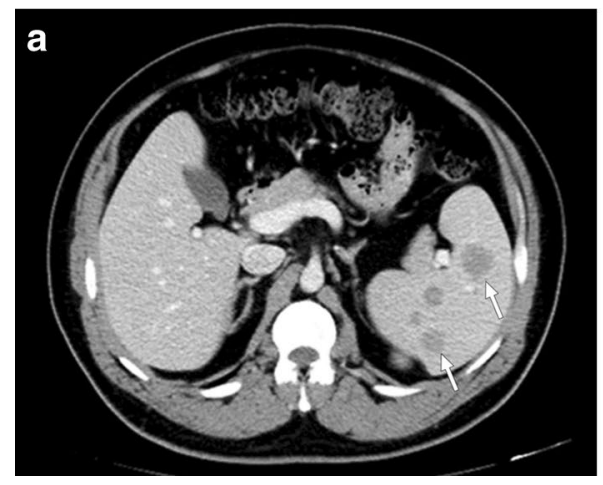

Fig. 28 Histopathologically proven splenic metastases in two different patients: a 35-year-old female patient with newly diagnosed cutaneous malignant melanoma. Initial staging contrast-enhanced abdominal CT showed multiple hypodense nodules in the spleen (arrows). US-guided percutaneous biopsy confirmed metastatic disease. b

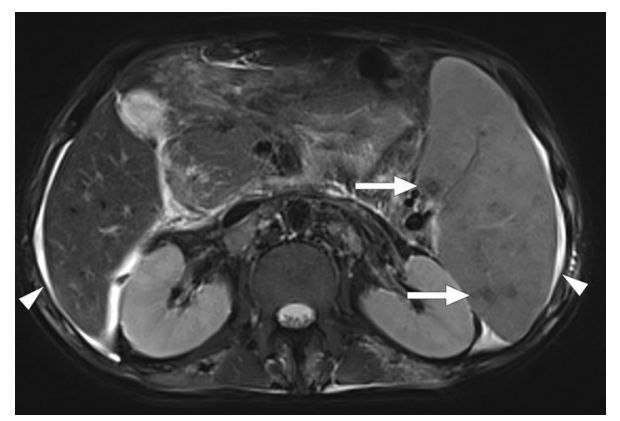

Fig. 29 Still's disease: 34-year-old male patient with no prior significant medical history now presented with fever, rash, polyarthralgia, and diffuse abdominal pain. Physical examination revealed severe splenomegaly. Initial US evaluation detected multiple hypoechoic parenchymal nodules in the spleen (not shown). Subsequent MRI exam showed multiple T2-hypointense splenic nodules (arrows) with associated splenomegaly. Also, note was made of a trace amount of intraperitoneal fluid (arrowhead). Extensive diagnostic workup diagnosed Still's disease

gadolinium-enhanced T1W images can be used to increase the lesion conspicuity (Fig. 30) [8].

\section{Predominantly cystic lesions}

In contrast to subcentimeter multiple parenchymal nodules detected during opportunistic fungal infections, pyogenic bacterial abscesses mostly cause solitary, large-sized cysts [1]. Other less common causes of splenic bacterial abscesses are melioidosis, actinomycosis, and nocardiosis, and these abscesses may also appear similar to ordinary pyogenic splenic abscesses. Hydatid cysts should also be kept in mind in the differential diagnosis of splenic cystic lesions, especially in endemic parts of the world. Benign cystic lesions of the spleen (epidermoid cyst, secondary splenic cysts)

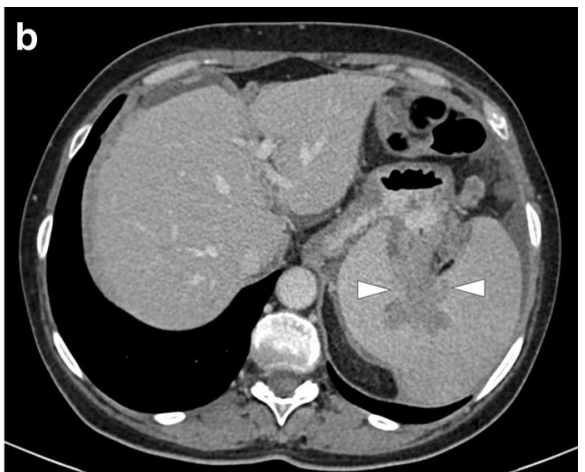

55-year-old female patient presenting with widespread peritoneal carcinomatosis. Axial plane post-contrast abdominal CT showed an infiltrative mass located in the splenic hilum (arrowheads) with gastric wall invasion. First-look surgery, with associated splenectomy, confirmed papillary serous tumor of peritoneal surfaces

(Fig. 31), chronic hematoma (Fig. 32), and lymphangioma may also be considered in the differential diagnoses [87, 88].

Although they are very rare, primary splenic angiosarcomas may present with bulky heterogeneous masses that contain large cystic areas. The thick contrast-enhancing irregular septa, the extension of the mass beyond the confines of the splenic parenchyma with associated nodular implants, and the presence of mural nodules should be alarming signs for malignancy over an infectious process (Fig. 33) [83].

\section{Mass-forming solid parenchymal lesions}

Infectious processes causing solid splenic masses have been reported in rare circumstances [2]. EBV-associated pseudotumors, tuberculomas, and rarely splenic pneumocystosis can be observed as solid lesions. Imaging findings are generally non-specific, and the differential diagnosis list is quite wide in the presence of solid lesions in the spleen. Primary benign and malignant neoplastic splenic diseases, lymphomatous involvement, extramedullary hematopoiesis (EMH), Gaucher nodules, and metastases are among the main differential diagnoses $[87,89]$.

Caution should be taken in terms of malignant lesions of the spleen (primary or metastases) in the presence of malignancy in other organs or splenic lesions with extrasplenic extension. Hamartoma, angiosarcoma, hemangioendothelioma, and Castleman disease mainly present with hypervascular splenic masses, while hypervascularity is generally not expected in sclerosing angiomatoid nodular transformation (SANT), lymphoma, metastases, EMH, infarctions, and infections [87]. "Spoke wheel" enhancement with a central stellate scar is a finding that may be suggestive of SANT [76, 77]. T2-hypointensity can be seen in SANT and sometimes lymphoma, whereas hamartomas and metastases are generally expected to be T2W hyperintense $[6-8,76]$. Splenic 


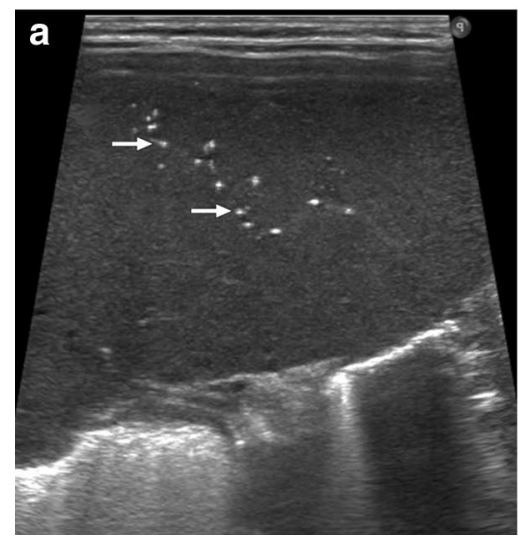

Fig. 30 Gamna-Gandy bodies in two different patients: a 6-monthold girl with a history of recurrent blood transfusions. Longitudinaloblique plane gray-scale US image showed multiple tiny echogenic foci (arrows). Considering the clinical information, imaging findings were found to be consistent with Gamna-Gandy nodules. b 50-year-

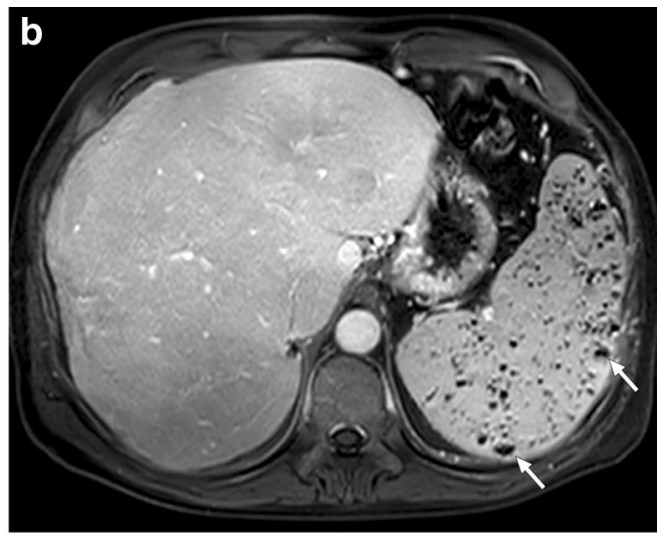

old male patient with known long-standing paroxysmal nocturnal hemoglobinuria and Budd-Chiari syndrome. Axial plane T1W postcontrast abdominal MR image showed innumerable Gamna-Gandy nodules (arrows) within the splenic parenchyma
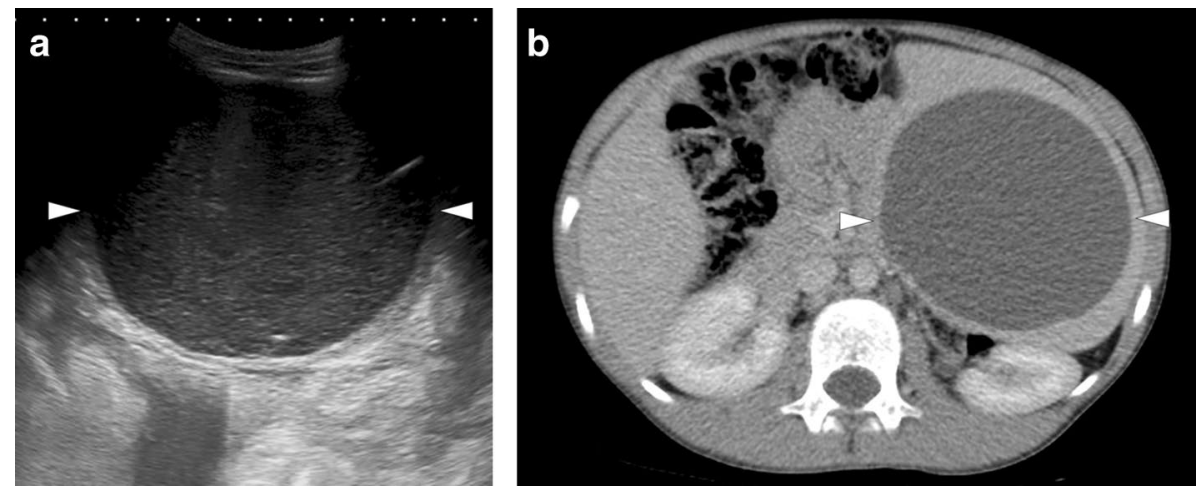

Fig. 31 Splenic epidermoid cyst: 11-year-old male patient presented with left upper quadrant pain. a Gray-scale US image showed a thinwalled cystic lesion (arrowheads) with low-level internal echoes. b Axial plane postcontrast abdominal CT image demonstrated the same lesion with well-defined borders and no apparent solid component (arrowheads). The imaging, clinical, and laboratory features of this lesion were considered to be consistent with a splenic epidermoid cyst. The patient was treated with percutaneous drainage and cavity sclerosis for symptomatic relief

non-infectious diseases affecting the spleen. Splenic infarction is observed as a peripheral, wedge-shaped nonenhancing area on CT and MR studies, whereas splenic rupture may present with different combinations of parenchymal laceration, intraparenchymal, sub-capsular, and/or intraperitoneal hemorrhage.

In general, infectious diseases causing splenomegaly may lead to splenic infarction and rupture, such as mononucleosis, malaria, leishmaniasis, babesiosis, and melioidosis. Portal hypertension and lymphoproliferative diseases are relatively more common causes of these two complications than infectious etiology [92, 93].

Recently, splenic infarcts have been reported in multisystem inflammatory syndrome in children (MIS-C). This disease should be considered in the differential diagnosis

Splenic infarction and spontaneous splenic rupture may develop in the course of many infectious and 


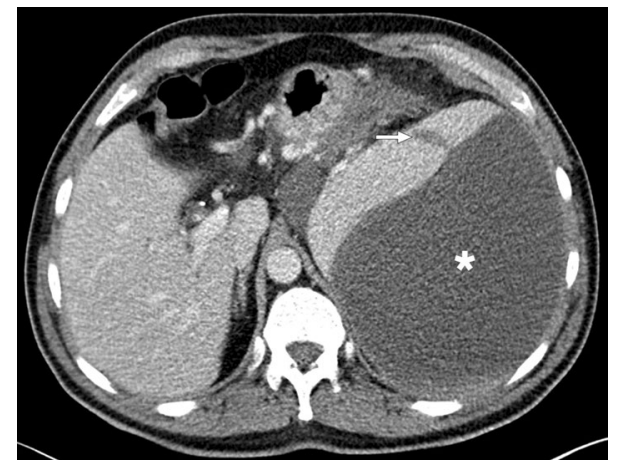

Fig. 32 Chronic hematoma: 47-year-old male patient with a history of non-traumatic splenic rupture due to chronic pancreatitis. Axial plane postcontrast abdominal follow-up CT image shows a large cystic splenic lesion in the subcapsular region (asterisk). Note was also made of a hypodense transparenchymal line corresponding to splenic laceration (arrow). US-guided percutaneous drainage of this cystic lesion confirmed chronic hematoma

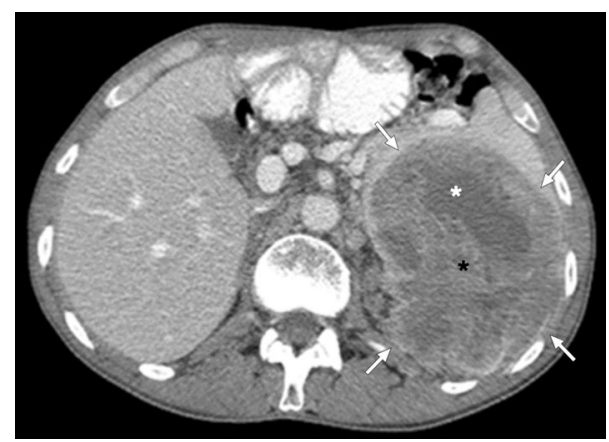

Fig. 33 Splenic angiosarcoma: 25-year-old male patient presented with relatively recent onset fever, weight loss, palpitations, and localized left upper quadrant abdominal pain. Axial plane postcontrast abdominal CT showed a large, heterogeneously-enhancing, complexappearing mass (arrows) with cystic (white asterisk) and solid (black asterisk) components. The lesion was also extending beyond the confines of the splenic parenchyma. Findings were found to be highly concerning for a primary splenic malignancy, and the patient underwent splenectomy. Final histopathological examination confirmed splenic angiosarcoma

of children with splenic infarcts of unknown etiology in these times of the COVID-19 pandemia (Fig. 36) [94].

\section{Calcifications}

Scattered splenic parenchymal calcifications are mostly expected to develop in the chronic stage of granulomatous diseases. These lesions may be seen in brucellosis, mycobacterial and fungal infections (Fig. 37). The presence of widespread parenchymal calcification in a shrunken spleen is a well-known imaging finding in patients with sickle cell anemia before autosplenectomy (Fig. 38). Diffuse splenic calcifications may also be observed in patients with amyloidosis, systemic lupus erythematosus (SLE), and treated Hodgkin lymphoma [95].

Focal calcifications may develop in the hydatid cyst wall and within the solid matrix at a later stage. Pneumocystosis, although rare, may present with annular calcifications. Wall calcifications can also be seen in non-infectious benign cystic lesions such as congenital splenic cysts or secondary splenic cysts. Furthermore, advanced stages of splenic infarctions and benign solid lesions such as hamartoma or hemangioma may contain intralesional calcifications [95].

\section{Conclusion}

The spleen is the largest lymphoid organ in the body and is a common site of infection. Imaging findings of different pathogenic agents may be similar. However, certain radiological patterns could be suggestive for certain agents. Awareness of these patterns together with differential diagnoses may have a significant impact on the management of the patient. Additionally, it should be kept in mind that clinical history and laboratory workup are crucial for correct diagnosis in the imaging evaluation of splenic infections.

Despite the absence of formal studies in the literature to guide differential diagnosis of splenic infections from various benign and malignant disorders, several imaging features mentioned in this manuscript, in addition to detailed clinical history and serological findings, may facilitate the diagnosis of some splenic infections. As imaging plays a fundamental role in recognizing infection, imagers should be familiar with potential imaging findings and pitfalls. However, it should be borne in mind that histopathological confirmation can be made in cases where imaging findings are equivocal. 

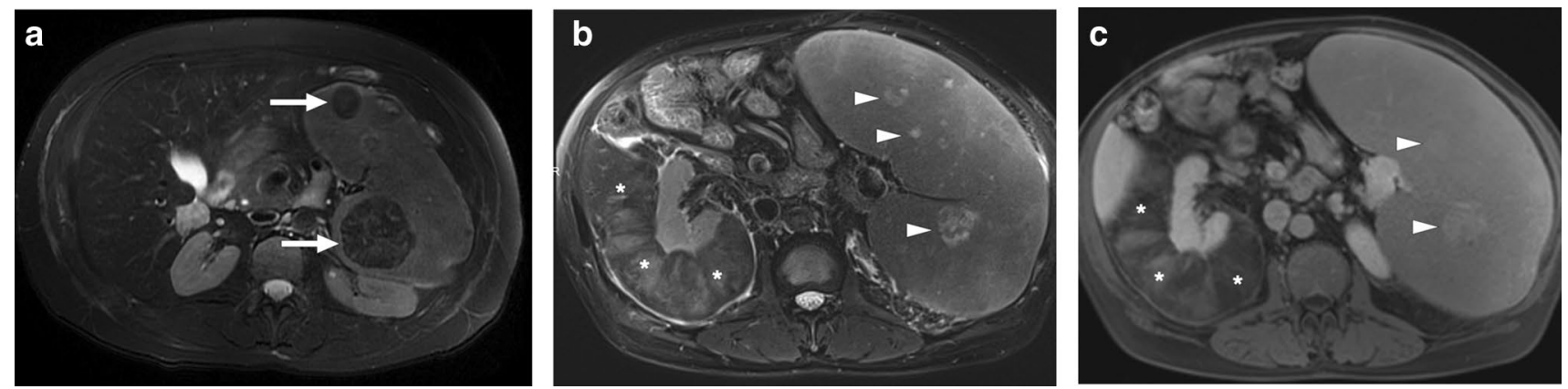

Fig. 34 Extramedullary hematopoiesis (EMH) of the spleen in two different patients: a 63-year-old female patient with a long-standing history of chronic myeloid leukemia. Axial plane T2W-fat-suppressed MR image shows hypointense EMH nodules. Their hypointensity is likely related to iron deposition in the chronic stage. These lesions were stable over seven years of follow-up (arrows). b and c 57-yearold male patient with primary myelofibrosis. b Axial plane T2Wfat-suppressed shows hyperintense lesions within the spleen (arrow-

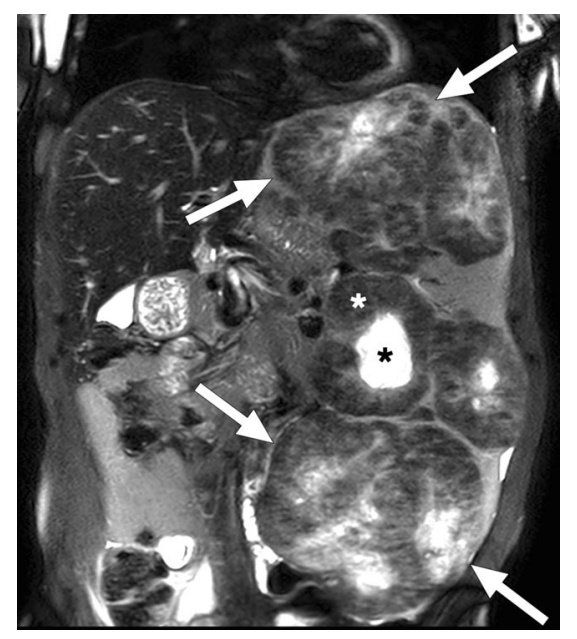

Fig. 35 Splenic Gaucheroma. 51-year-old male patient with known long-standing Gaucher disease presented with abdominal pain. Coronal plane T2W-fat-suppressed abdominal MR image showed multiple splenic masses (arrows) with hypointense siderotic walls (white asterisks) and hyperintense cystic centers (black asterisks). Note was also made of massive splenomegaly. US-guided percutaneous biopsy of these masses confirmed Gaucheromas heads). c T1W postcontrast axial plane MR image demonstrated contrast enhancement within these nodules (arrowheads). There was also a large right perirenal mass almost completely encircling the right kidney (asterisks). Percutaneous biopsy of the right perirenal lesion was consistent with extramedullary hematopoiesis. Based on this histopathologic finding, the splenic lesions were also considered to represent EMH foci. The lesions are stable over two years of imaging follow-up

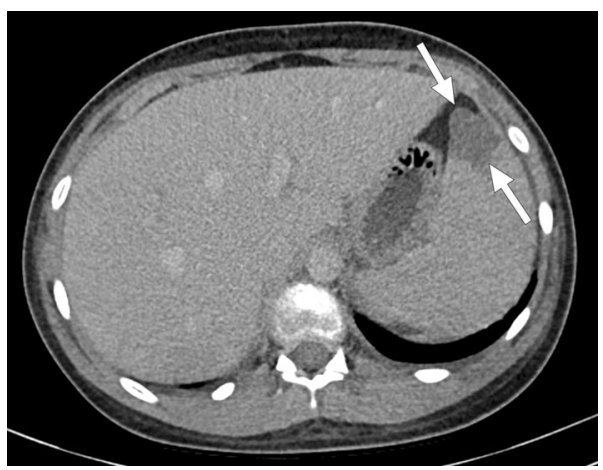

Fig. 36 MIS-C: 12-year-old boy with a recent history of COVID-19 infection presented to the ER with complaints of fever, malaise, rash, abdominal pain, and vomiting. Physical examination was unremarkable except for mildly enlarged cervical lymph nodes. Thoracoabdominal CT was ordered in order to rule out possible infectious foci. Axial plane postcontrast abdominal CT showed small-sized parenchymal infarcts within the spleen (arrows). The patient was hospitalized immediately after this CT study. Thorough clinical and laboratory evaluation led to the diagnosis of MIS-C 


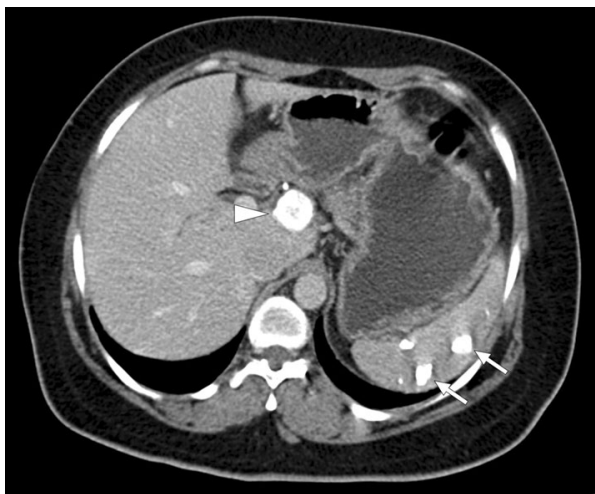

Fig. 37 Calcified splenic granulomas: 41-year-old female patient with a known remote history of pulmonary tuberculosis. Axial plane postcontrast abdominal CT showed multiple calcified nodular lesions in the spleen (arrows), which were thought to represent calcified splenic granulomas. Also, note was made of a calcified lymph node at the liver hilum (arrowhead)

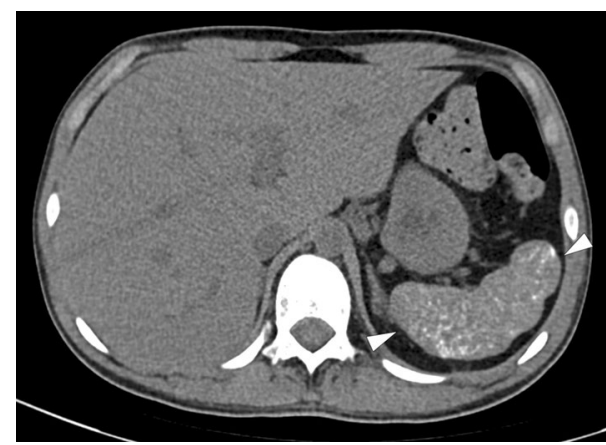

Fig. 38 Sickle cell anemia: 19-year-old male patient with known sickle cell anemia. Axial plane non-enhanced abdominal CT showed diffuse punctate calcifications within the parenchyma of the partially shrunken spleen (arrowheads)

Author contributions ADK, AU and $\mathrm{OO}$ wrote the manuscript. $\mathrm{PFH}$, $\mathrm{DA}, \mathrm{MNO}$ and MK provided the cases and edited the text. All of the authors read and approved the final manuscript.

Funding No funding was received for this project.

\section{Declaration}

Conflict of interest The authors declare that they have no conflict of interest.

\section{References}

1. Chun JY, Kim YH. Spleen Infectious and Inflammatory Disorders. In: Hamm B, Ros PR, editors. Abdominal Imaging. Berlin, Heidelberg: Springer Berlin Heidelberg; 2013. p. 1511-22.
2. Gaetke-Udager K, Wasnik AP, Kaza RK, Al-Hawary MM, Maturen KE, Udager AM, et al. Multimodality imaging of splenic lesions and the role of non-vascular, image-guided intervention. Abdominal imaging. 2014;39(3):570-87.

3. Vancauwenberghe T, Snoeckx A, Vanbeckevoort D, Dymarkowski S, Vanhoenacker FM. Imaging of the spleen: what the clinician needs to know. Singapore medical journal. 2015;56(3):133.

4. Sammon J, Twomey M, Crush L, Maher MM, O'Connor OJ, editors. Image-guided percutaneous splenic biopsy and drainage. Seminars in interventional radiology; 2012: Thieme Medical Publishers.

5. Karlo CA, Stolzmann P, Do RK, Alkadhi H. Computed tomography of the spleen: how to interpret the hypodense lesion. Insights into imaging. 2013;4(1):65-76.

6. Palas J, Matos AP, Ramalho M. The spleen revisited: an overview on magnetic resonance imaging. Radiology Research and Practice. 2013;2013.

7. Elsayes KM, Narra VR, Mukundan G, Lewis Jr JS, Menias CO, Heiken JP. MR imaging of the spleen: spectrum of abnormalities. Radiographics. 2005;25(4):967-82.

8. Luna A, Ribes R, Caro P, Luna L, Aumente E, Ros PR. MRI of focal splenic lesions without and with dynamic gadolinium enhancement. American Journal of Roentgenology. 2006;186(6):1533-47.

9. Bittencourt LK, Matos C, Coutinho AC. Diffusion-weighted magnetic resonance imaging in the upper abdomen: technical issues and clinical applications. Magnetic Resonance Imaging Clinics. 2011;19(1):111-31.

10. Jang KM, Kim SH, Hwang J, Lee SJ, Kang TW, Lee MW, et al. Differentiation of malignant from benign focal splenic lesions: added value of diffusion-weighted MRI. American Journal of Roentgenology. 2014;203(4):803-12.

11. Li Y, George A, Arnaout S, Wang JP, Abraham GM, editors. Splenic infarction: an under-recognized complication of infectious mononucleosis? Open forum infectious diseases; 2018: Oxford University Press US.

12. Jenson HB. Acute complications of Epstein-Barr virus infectious mononucleosis. Current opinion in pediatrics. 2000;12(3):263-8.

13. Custer RP, Smith EB. The pathology of infectious mononucleosis. Blood. 1948;3(8):830-57.

14. Garten AJ, Mendelson DS, Halton KP. CT manifestations of infectious mononucleosis. Clinical imaging. 1992;16(2):114-6.

15. Rosenbaum L, Fekrazad MH, Rabinowitz I, Vasef MA. EpsteinBarr virus-associated inflammatory pseudotumor of the spleen: report of two cases and review of the literature. Journal of hematopathology. 2009;2(2):127-31.

16. Van Baeten C, Van Dorpe J. Splenic Epstein-Barr Virus-Associated Inflammatory Pseudotumor. Archives of pathology \& laboratory medicine. 2017;141(5):722-7.

17. Maeda E, Akahane M, Kiryu S, Kato N, Yoshikawa T, Hayashi N, et al. Spectrum of Epstein-Barr virus-related diseases: a pictorial review. Japanese journal of radiology. 2009;27(1):4-19.

18. Paris S, Weiss S, Ayers Jr W, Clarke L. Splenic abscess. The American surgeon. 1994;60(5):358-61.

19. P. N. Infectious Diseases and Biologic Weapons. In: LA F, editor. Anesthesia and Uncommon Diseases Sixth ed. Philadelphia: W.B. Saunders; 2012. p. 369-400.

20. Ayyala RS, Anupindi SA, Taylor GA, Gee MS, Bellah RD, Chauvin NA. Imaging of Splenic Infections (and Their Mimickers) in Children. Current Radiology Reports. 2016;4(1):2.

21. Thanos L, Dailiana T, Papaioannou G, Nikita A, Koutrouvelis H, Kelekis D. Percutaneous CT-guided drainage of splenic abscess. American Journal of Roentgenology. 2002;179(3):629-32. 
22. Al-Nakshabandi NA. The spectrum of imaging findings of brucellosis: a pictorial essay. Canadian Association of Radiologists Journal. 2012;63(1):5-11.

23. Sayilir K, Iskender G, Ogan MC, Erdil F. Splenic abscess due to brucellosis. The Journal of Infection in Developing Countries. 2008;2(05):394-6.

24. Torres US, Cardoso LV, D'Ippolito G. A pathognomonic calcification pattern in chronic splenic brucellosis. Brazilian Journal of Infectious Diseases. 2015;19(6):664-5.

25. Lin S-F, Zheng L, Zhou L. Solitary splenic tuberculosis: a case report and review of the literature. World journal of surgical oncology. 2016;14(1):1-4.

26. Rhazal F, Lahlou M, Benamer S, Daghri J, Essadel E, Mohammadine E, et al., editors. Splenomegaly and splenic pseudotumor due to tuberculosis: six new cases. Annales de chirurgie; 2004.

27. Chandra S, Srivastava D, Gandhi D. Splenic tuberculosis: an unusual sonographic presentation. International journal of clinical practice. 1999;53(4):318-9.

28. Kandhasamy SC, Elamurugan T, Naik D, Rohith G, Ramakrishnaiah VPN. Systemic Melioidosis With Ruptured Splenic Abscess. Cureus. 2020;12(5).

29. Chen H, Hu Z-q, Fang Y, Lu X-x, Li L-d, Li Y-l, et al. A case report: Splenic abscess caused by Burkholderia pseudomallei. Medicine. 2018;97(26).

30. Wiersinga WJ, Virk HS, Torres AG, Currie BJ, Peacock SJ, Dance DA, et al. Melioidosis. Nature reviews Disease primers. 2018;4(1):1-22.

31. Wibulpolprasert B, Dhiensiri T. Visceral organ abscesses in melioidosis: sonographic findings. Journal of clinical ultrasound. 1999;27(1):29-34.

32. Liston TE, Koehler JE. Granulomatous hepatitis and necrotizing splenitis due to Bartonella henselae in a patient with cancer: case report and review of hepatosplenic manifestations of Bartonella infection. Clinical infectious diseases. 1996;22(6):951-7.

33. Gilad J, Wolak A, Borer A, Benharroch D, Avidor B, Giladi M, et al. Isolated splenic cat scratch disease in an immunocompetent adult woman. Clinical Infectious Diseases. 2003;36(1):e10-e3.

34. Danon O, Duval-Arnould M, Osman Z, Boukobza B, Kazerouni F, Cadranel J-F, et al. Hepatic and splenic involvement in cat-scratch disease: imaging features. Abdominal imaging. 2000;25(2):182-3.

35. Hopkins KL, Simoneaux SF, Patrick LE, Wyly JB, Dalton MJ, Snitzer JA. Imaging manifestations of cat-scratch disease. AJR American journal of roentgenology. 1996;166(2):435-8.

36. Saad E, Elsamani E, Abdelrahman W. Spontaneous splenic rupture complicating severe P. falciparum infection: a case report and literature review. Case reports in infectious diseases. 2019;2019.

37. Organization WH. Guidelines for malaria vector control: World Health Organization; 2019.

38. Cinquetti G, Banal F, Rondel C, Plancade D, de Saint Roman C, Adriamanantena D, et al. Splenic infarction during Plasmodium ovale acute malaria: first case reported. Malaria journal. 2010;9(1):1-3.

39. Hershey FB, Lubitz JM. Spontaneous rupture of the malarial spleen: case report and analysis of 64 reported cases. Annals of surgery. 1948;127(1):40.

40. Osman MF, Elkhidir IM, Rogers Jr SO, Williams M. Non-operative management of malarial splenic rupture: the Khartoum experience and an international review. International Journal of Surgery. 2012;10(9):410-4.

41. Nadeem Kassam SM, Hameed K, Ali A, Surani S. Case Report: Sudden Splenic Rupture in a Plasmodium falciparum-Infected Patient. International Journal of General Medicine. 2020;13:595.

42. Lee LK, Hahn PF. Beyond splenomegaly: An image-based review of infectious and inflammatory diseases of the spleen. Applied radiology. 2017;46(7):24-9.
43. Ameur HB, Affes N, Abdelhedi C, Kchaou A, Boujelbene S, Beyrouti MI. Hydatid cyst of the spleen: tunisian series of 21 cases. Indian Journal of Surgery. 2015;77(2):515-9.

44. Rasheed K, Zargar SA, Telwani AA. Hydatid cyst of spleen: a diagnostic challenge. North American journal of medical sciences. 2013;5(1):10.

45. Pukar M, Pukar SM. Giant solitary hydatid cyst of spleenA case report. International journal of surgery case reports. 2013;4(4):435-7.

46. Akhan O, Akkaya S, Dağoğlu MG, Akpınar B, Erbahçeci A, Çiftçi $\mathrm{T}$, et al. Percutaneous treatment of splenic cystic echinococcosis: results of 12 cases. Cardiovascular and interventional radiology. 2016;39(3):441-6.

47. Örmeci N, Kalkan Ç, Karakaya F, Erden A, Köse KS, Tüzün AE, et al. Percutaneous treatment with the Örmeci technique for hydatid disease located in the spleen: Single center experience for twenty six years. The Turkish Journal of Gastroenterology. 2018;29(5):566.

48. Dasgupta S, Saha M, Chakrabarti S, Chakraborty J. Visceral leishmaniasis with pleural effusion in an immunocompetent patient. Lung India: Official organ of Indian Chest Society. 2014;31(1):56.

49. Mao G, Yang G, Cheng Y, Zee CS, Huang W, Ni W, et al. Multiple nodular lesions in spleen associated with visceral leishmaniasis: a case report of MRI-findings. Medicine. 2014;93(29).

50. Vannier E, Krause PJ. Human babesiosis. New England Journal of Medicine. 2012;366(25):2397-407.

51. Mojtahed A, Bates D, Hahn P. Splenic findings in patients with acute babesiosis. Abdominal Radiology. 2020;45(3):710-5.

52. Kuwayama DP, Briones RJ. Spontaneous splenic rupture caused by Babesia microti infection. Clinical Infectious Diseases. 2008;46(9):e92-e5.

53. Li S, Goyal B, Cooper JD, Abdelbaki A, Gupta N, Kumar Y. Splenic rupture from babesiosis, an emerging concern? A systematic review of current literature. Ticks and tick-borne diseases. 2018;9(6):1377-82.

54. Patel KM, Johnson JE, Reece R, Mermel LA. Babesiosis-associated splenic rupture: case series from a hyperendemic region. Clinical Infectious Diseases. 2019;69(7):1212-7.

55. Hung Y-M, Kao C-H, Wann S-R, Wang PY-P, Chang Y-T. Disseminated Nocardiosis with Lung, Liver and Spleen Abscesses in Sweet Syndrome: A Case Report. Journal of Acute Medicine. 2017;7(4):167.

56. Lederman ER, Crum NF. A case series and focused review of nocardiosis: clinical and microbiologic aspects. Medicine. 2004;83(5):300-13

57. Vanhoenacker F, De Backer A, de Beeck BO, Maes M, Van Altena R, Van Beckevoort D, et al. Imaging of gastrointestinal and abdominal tuberculosis. European Radiology Supplements. 2004;14(3):E103-E15.

58. Clark H, Khatri G, Kapur P, Pedrosa I. Splenic Involvement in Disseminated Mycobacterium avium-intracellulare Infection: Magnetic Resonance Imaging Findings. Journal of computer assisted tomography. 2018;42(1):151-4.

59. Chung MJ, Lee KS, Koh W-J, Lee JH, Kim TS, Kwon OJ, et al. Thin-section CT findings of nontuberculous mycobacterial pulmonary diseases: comparison between Mycobacterium aviumintracellulare complex and Mycobacterium abscessus infection. Journal of Korean medical science. 2005;20(5):777.

60. O'Neal CB, Ball SC. Splenic pneumocystosis: an atypical presentation of extrapulmonary Pneumocystis infection. The AIDS reader. 2008;18(10):503-.

61. Walzer PD, Perl DP, Krogstad DJ, Rawson PG, Schultz MG. Pneumocystis carinii pneumonia in the United States: epidemiologic, diagnostic, and clinical features. Annals of internal medicine. 1974;80(1):83-93. 
62. Abbas H, Patel H, Baiomi A, Niazi M, Vakde T, Chilimuri S. A rare case of splenic pneumocystis jirovecii in a HIV-Positive Patient. Case reports in gastrointestinal medicine. 2020;2020.

63. Wong VK, Turmezei T, Weston V. Actinomycosis. Bmj. 2011;343.

64. Wang H-K, Sheng W-H, Hung C-C, Chen Y-C, Liew P-L, Hsiao $\mathrm{C}-\mathrm{H}$, et al. Hepatosplenic actinomycosis in an immunocompetent patient. Journal of the Formosan Medical Association. 2012;111(4):228-31.

65. Laniado-Laborin R. Expanding understanding of epidemiology of coccidioidomycosis in the Western hemisphere. Annals of the New York Academy of Sciences. 2007;1111(1):19-34.

66. Bird GR, Libke RD, Billelo JF, Parks NA, Pollard JS. Disseminated coccidioidomycosis with clinically evident splenomegaly in an immunocompetent host, first case reported in the literature. McGill Journal of Medicine: MJM. 2009;12(2).

67. Musil IL, Gilbertson-Dahdal D, Elliott SP. Disseminated coccidioidomycosis in an immunodeficient 16-year-old female. Radiology case reports. 2008;3(4):237.

68. Kauffman CA. Histoplasmosis: a clinical and laboratory update. Clinical microbiology reviews. 2007;20(1):115-32.

69. Ashraf N, Kubat RC, Poplin V, Adenis AA, Denning DW, Wright $\mathrm{L}$, et al. Re-drawing the maps for endemic mycoses. Mycopathologia. 2020:1-23.

70. Kim DY, Kim Y, Baek SY, Yoon H-K. Simultaneous thoracic and abdominal presentation of disseminated cryptococcosis in two patients without HIV infection. American Journal of Roentgenology. 2003;181(4):1055-7.

71. Ergene U, Akcali Z, Ozbalci D, Nese N, Senol S. Disseminated aspergillosis due to Aspergillus niger in immunocompetent patient: a case report. Case reports in infectious diseases. 2013;2013.

72. Yeom SK, Kim HJ, Byun JH, Kim AY, Lee M-G, Ha HK. Abdominal aspergillosis: CT findings. European journal of radiology. 2011;77(3):478-82.

73. Chapman J, Bansal P, Goyal A, Azevedo AM. Splenomegaly. StatPearls [Internet]. 2020.

74. Bottieau E, Clerinx J, Van den Enden E, Van Esbroeck M, Colebunders R, Van Gompel A, et al. Infectious Mononucleosis-Like Syndromes in Febrile Travelers Returning From the Tropics. Journal of travel medicine. 2006;13(4):191-7.

75. Pozo AL, Godfrey EM, Bowles KM. Splenomegaly: investigation, diagnosis and management. Blood reviews. 2009;23(3):105-11.

76. Thut D, Smolinski S, Morrow M, Shirley McCarthy M, Alsina J, Kreychman A, et al. A diagnostic approach to splenic lesions. Applied Radiology. 2017;46(2):7.

77. Karaosmanoglu DA, Karcaaltincaba M, Akata D. CT and MRI findings of sclerosing angiomatoid nodular transformation of the spleen: spoke wheel pattern. Korean journal of radiology. 2008;9(Suppl):S52.

78. Melchionda F, Varani S, Carfagnini F, Belotti T, Di Muccio T, Tigani R, et al. Spleen nodules: a potential hallmark of Visceral Leishmaniasis in young children. BMC infectious diseases. 2014;14(1):1-5.

79. Warshauer DM, editor Splenic sarcoidosis. Seminars in Ultrasound, CT and MRI; 2007: Elsevier.

80. Saboo S, Krajewski K, O'regan K, Giardino A, Brown J, Ramaiya N, et al. Spleen in haematological malignancies: spectrum of imaging findings. The British journal of radiology. 2012;85(1009):81-92.
81. Ozeki M, Fukao T. Generalized lymphatic anomaly and GorhamStout disease: overview and recent insights. Advances in wound care. 2019;8(6):230-45.

82. Patti R, Iannitto E, Di Vita G. Splenic lymphangiomatosis showing rapid growth during lactation: a case report. World journal of gastroenterology: WJG. 2010;16(9):1155.

83. Abbott RM, Levy AD, Aguilera NS, Gorospe L, Thompson WM. From the archives of the AFIP: primary vascular neoplasms of the spleen: radiologic-pathologic correlation. Radiographics. 2004;24(4):1137-63.

84. Louis TH, Sanders JM, Stephenson JS, Harbour LN, Ford III KL, editors. Splenic hemangiomatosis. Baylor University Medical Center Proceedings; 2011: Taylor \& Francis.

85. Assimakopoulos SF, Karamouzos V, Papakonstantinou C, Zolota V, Labropoulou-Karatza C, Gogos C. Granulomas formation in lymph nodes, liver and spleen in adult-onset Still's disease: A case report. Journal of clinical medicine research. 2013;5(2):144.

86. Bhatt S, Simon R, Dogra VS. Gamna-Gandy bodies: sonographic features with histopathologic correlation. Journal of ultrasound in medicine. 2006;25(12):1625-9.

87. Lee H-j, Kim JW, Hong JH, Kim GS, Shin SS, Heo SH, et al. Cross-sectional imaging of splenic lesions: RadioGraphics fundamentals online presentation. RadioGraphics. 2018;38(2):435-6.

88. Urrutia M, Mergo PJ, Ros LH, Torres GM, Ros PR. Cystic masses of the spleen: radiologic-pathologic correlation. Radiographics. 1996;16(1):107-29.

89. Tseng S-Y, Niu D-M, Chu T-H, Yeh Y-C, Huang M-H, Yang T-F, et al. Very rare condition of multiple Gaucheroma: A case report and review of the literature. Molecular genetics and metabolism reports. 2019;20:100473.

90. Regenboog M, Bohte AE, Somers I, van Delden OM, Maas M, Hollak CE. Imaging characteristics of focal splenic and hepatic lesions in type 1 Gaucher disease. Blood Cells, Molecules, and Diseases. 2016;60:49-57.

91. Regenboog M, Bohte AE, Akkerman EM, Stoker J, Hollak CE. Iron storage in liver, bone marrow and splenic Gaucheroma reflects residual disease in type 1 Gaucher disease patients on treatment. British journal of haematology. 2017;179(4):635-47.

92. Lam GY, Chan AK, Powis JE. Possible infectious causes of spontaneous splenic rupture: a case report. Journal of medical case reports. 2014;8(1):1-5.

93. Im JH, Chung M-H, Lee H-J, Kwon HY, Baek JH, Jang J-H, et al. Splenic infarction and infectious diseases in Korea. BMC Infectious Diseases. 2020;20(1):1-7.

94. Hameed S, Elbaaly H, Reid CE, Santos RM, Shivamurthy V, Wong $\mathrm{J}$, et al. Spectrum of Imaging Findings at Chest Radiography, US, $\mathrm{CT}$, and MRI in Multisystem Inflammatory Syndrome in Children Associated with COVID-19. Radiology. 2021;298(1):E1-E10.

95. Consul N, Javed-Tayyab S, Lall C, Jensen CT, Menias CO, Pickhardt PJ, et al. Calcified splenic lesions: pattern recognition approach on CT with pathologic correlation. American Journal of Roentgenology. 2020;214(5):1083-91.

Publisher's Note Springer Nature remains neutral with regard to jurisdictional claims in published maps and institutional affiliations. 